\title{
Formation and Stabilization of Multiple Water-in-Water-in-Water (W/W/W) emulsions
}

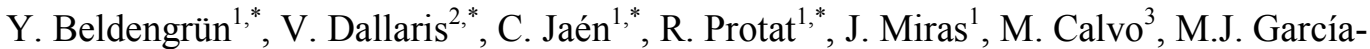 \\ Celma $^{2}$, J. Esquena ${ }^{1 ; \#}$ \\ ${ }^{1}$ Institute of Advanced Chemistry of Catalonia, Spanish National Research Council (IQAC-CSIC) and \\ Networking Research Center on Bioengineering, Biomaterials and Nanomedicine (CIBER-BBN), \\ Barcelona, 08034 Spain \\ ${ }^{2}$ Faculty of Pharmacy and Food Science, University of Barcelona, Diagonal Campus \\ Joan XXIII, 27-31, Barcelona, 08028, Spain \\ 3 Advanced Optical Microscopy Unit. Scientific and Technological Centers of the University of \\ Barcelona, Faculty of Medicine, Barcelona, Spain
}

* These co-authors equally contributed to this work, each one developing a different aspect.

\# Corresponding author: jordi.esquena@iqac.csic.es

\begin{abstract}
Multiple Water-in-Water-in-Water (W/W/W) emulsions have been prepared, stabilized and characterized. The main objective has been to find a simple and low-cost method for the preparation of W/W/W emulsions. The system composed of gelatin, maltodextrin and water has been used, and two different methods have been studied for producing multiple emulsions in this system. In the first method, maltodextrin-in-gelatin (M/G) emulsions with small droplet size were formed by $\mathrm{pH}$-induced nucleation of maltodextrin droplets, and afterwards, maltodextrinin-gelatin-in-maltodextrin $(\mathrm{M} / \mathrm{G} / \mathrm{M})$ multiple emulsions were obtained by dispersing $\mathrm{M} / \mathrm{G}$ droplets into maltodextrin solutions. The second method consisted in cooling down gelatin-inmaltodextrin $(\mathrm{G} / \mathrm{M})$ emulsions, leading to the spontaneous formation of inner maltodextrin droplets. The latter method allowed producing more homogenous $\mathrm{M} / \mathrm{G} / \mathrm{M}$ multiple emulsion droplets. The colloidal stability of such emulsions greatly improved with the addition of mucin particles, which is a glycoprotein that adsorbs on the $\mathrm{G} / \mathrm{M}$ interface. Stable $\mathrm{M} / \mathrm{G} / \mathrm{M}$ multiple emulsions have been prepared and characterized by fluorescence optical microscopy, where contrast has been enhanced through covalently labelling the various components with fluorescent dyes. To our knowledge, this is the first report of a simple and cost-effective method for the production of multiple $\mathrm{W} / \mathrm{W} / \mathrm{W}$ emulsions, without using microfluidic techniques. Moreover, the present work also demonstrates that mucin microparticles can be effective stabilizers for protein-in-polysaccharide emulsions, and these dispersions can be easily prepared by phase transition methods.
\end{abstract}

\section{Introduction}


Water-in-water (W/W) emulsions can be formed in aqueous mixtures with two equilibrium aqueous phases ${ }^{1}$. W/W emulsions have been known for very long time, since they were described for the first time by Beijerinck ${ }^{2}$ in 1896 , who observed the formation of droplets in cell cultures prepared with aqueous mixtures of gelatin and soluble starch. It is well known that aqueous mixtures containing two incompatible hydrophilic polymers can phase separate in two aqueous phases, in absence of both oil and surfactant. These systems are usually denoted as Aqueous Two-Phase Systems (ATPS) ${ }^{1,3}$, in which the two aqueous solutions are in thermodynamic equilibrium. Both phases contain water as the same solvent, but one phase is relatively rich in one solute, whereas the other phase is enriched with another solute.

A great variety of aqueous systems, with mixtures of proteins and polysaccharides, are known to produce phase separation in two aqueous phases ${ }^{4}$. This segregative phase separation occurs because of thermodynamic incompatibility between the charged protein and a neutral or similarly charged polysaccharide. The main factor that produces phase separation is the difference in hydration of the two macromolecules, which can be explained in terms of FloryHuggins interaction parameters, between the two polymers and their respective interactions with water ${ }^{6}$. Usually, the most charged polymer is highly hydrated, and it expels the less hydrated polymer. Another factor that can induce phase separation is the "excluded volume" or "steric exclusion" effect. It implies that, above a critical concentration of macromolecules, there is an osmotic attractive force that induces phase separation into two aqueous phases, each one enriched in one of the polymers ${ }^{7,8}$. Because of that, phase segregation is promoted by differences in molecular weight, and many systems that show aqueous phase segregation consist of a charged polymer with very high molecular weight, mixed with a non-charged polymer with lower molecular weight.

Nowadays, it is known that Aqueous Two-Phase Systems are commonly observed in mixtures of two hydrophilic polymers. The interface between two aqueous phases is not well defined, since bulk properties (diffraction index, density, dielectric constant, etc.) have very small variations from one phase to the other, across the interface. Consequently, interfacial regions in water-water interfaces tend to be relatively thick and fuzzy, in comparison to conventional oilwater interfaces. Typically, the interfacial region is thicker than the effective size of the polymers, and it is governed by a soft gradient in macroscopic properties. Recently, various authors have focused their researches on the special properties of water-water interfaces ${ }^{9-14}$, which often have an asymmetric distribution of ions across the interface.

Interfacial tensions are very low in Aqueous Two-Phase Systems ${ }^{15-19}$. This is not surprising, given that the two phases have water as the same solvent and the two compositions across the interface are quite similar. It is known that water-water interfacial tension is extremely low at the vicinity of the critical point of phase diagrams, and tension values lower than $10^{-3} \mathrm{mN} / \mathrm{m}$ have been measured. Moreover, these low values are not unusual in such systems, and it is also known that interfacial tension becomes virtually zero at the critical point, where the two aqueous phases merge and become one single phase. Because of such low interfacial tensions, small molecules do not adsorb on water-water interfaces, simply because their energy of adsorption is lower than $\mathrm{kT}$.

In recent years, the study of water-water interfaces and the stabilization of W/W emulsions has become a topic of remarkable interest ${ }^{1}$. Emulsification in Aqueous Two-Phase Systems allows 
the formation of water-in-water emulsions where droplets that contain most of one solute are dispersed in a continuous phase, which contains most of the second solute.

Currently, several authors have focused their attention on the formation of $\mathrm{W} / \mathrm{W}$ emulsions by microfluidic techniques, allowing a precise control of droplet size, with a very low polydispersity ${ }^{20-23}$. It has been shown that microfluidic formation of $\mathrm{W} / \mathrm{W}$ emulsions requires a quite low pressure ${ }^{21}$, which is related to the small interfacial tension of such systems. Moreover, microfluidics has allowed the formation of multiple emulsions in presence of different aqueous phases, such as $\mathrm{W} / \mathrm{O} / \mathrm{W}^{24}$ or $\mathrm{W} / \mathrm{W} / \mathrm{O}^{25}$.

The long-term stabilization of water-in-water emulsions is not a simple task. Conventional surfactants cannot stabilize $\mathrm{W} / \mathrm{W}$ interfaces, but many methods have been described for preparing and stabilizing $\mathrm{W} / \mathrm{W}$ emulsions with adsorbed particles on the interface, similarly to conventional Pickering emulsions. The work by Poortinga et al is credited as the first report that described the Pickering stabilization of $\mathrm{W} / \mathrm{W}$ emulsions, using various types of fat particles adsorbed on a $\mathrm{W} / \mathrm{W}$ interface ${ }^{26}$. Following this work, a great interest has been raised in recent years, and a wide variety of different particles have demonstrated to be effective in stabilizing $\mathrm{W} / \mathrm{W}$ emulsions ${ }^{27}$, which include latex particles $^{28,29}$, proteinaceous particles ${ }^{30-33}$, partially hydrophobized silica particles ${ }^{34,35}$, clay nanoplatelets ${ }^{36,37}$, cellulose nanorods ${ }^{38}$ or cellulose nanocrystals $^{39}$, as illustrative examples. The adsorption of particles on water-water interfaces and the stabilization of $\mathrm{W} / \mathrm{W}$ emulsions by a Pickering mechanism have been studied in detail by T. Nicolai and his coworkers ${ }^{29,39-42}$. Moreover, very recent results have demonstrated the usefulness of particle functionalization with polysaccharides, which can greatly enhance adsorption of particles on water-water interfaces ${ }^{43}$. Stability of W/W emulsions can be much improved by modulating the phase preference of the particles, because these emulsions are better stabilized if particles have a stronger preference for the continuous phase ${ }^{42}$. Consequently, particle-stabilized $\mathrm{W} / \mathrm{W}$ emulsions behave similarly to $\mathrm{O} / \mathrm{W}$ or $\mathrm{W} / \mathrm{O}$ conventional Pickering emulsions.

In any case, a great interest is focused on the use of biocompatible materials for the preparation and stabilization of $\mathrm{W} / \mathrm{W}$ emulsions. It should be remarked that $\mathrm{W} / \mathrm{W}$ emulsions can be formed using fully biocompatible and edible components ${ }^{44}$, For example, water/water phase separation often occurs in aqueous mixtures of proteins and polysaccharides ${ }^{4}$, at room temperature and neutral $\mathrm{pH}$, and these emulsions are very appropriate media for the encapsulation of living cells $^{33,45}$, water soluble drugs and biomolecules ${ }^{46}$. As water-in-water emulsions can be prepared with edible raw materials, these emulsions are of great interest in the formulation of fat-free and surfactant-free commercial products. Moreover, W/W emulsion might have enormous potential applications as novel fully-aqueous cosmetics or personal care products.

Different types of W/W emulsions have been described, including protein-in-polysaccharide and polysaccharide-in-protein emulsions. For example, Kasapis, Norton, Frith and their coauthors have studied in deep detail the gelatin-maltodextrin system ${ }^{15,47-49}$. They reported that the external phase tends to be the one with higher volume fraction, and thus, gelatin-in-maltodextrin emulsions can be prepared in maltodextrin-rich compositions, while maltodextrin-in-gelatin are formed in gelatin-rich formulations. Moreover, they reported that bicontinuous or multiple emulsions formed at intermediate compositions. It is also known that $\mathrm{W} / \mathrm{W}$ dispersions, formed in the gelatin-maltodextrin system, can be stable at room temperatures below the gelification point of gelatin, forming structured microgels. In a recent work, we have used gelatin microgels, 
obtained by crosslinking the dispersed phase of gelatin-in-maltodextrin emulsions, as carriers to encapsulate an enzyme, $\beta$-galactosidase ${ }^{46}$.

Multiple $\mathrm{O} / \mathrm{O} / \mathrm{O}$ emulsions are known and recent examples can be found in literature ${ }^{50}$. However, very little attention has been focused on water-in-water-in-water emulsions (W/W/W) emulsions $^{23,51,52}$. The existence of multiple $\mathrm{W} / \mathrm{W} / \mathrm{W}$, transiently observed in composition near $50 / 50$ volume fraction ratio, has been reported ${ }^{15,53}$. These emulsions, as well as bicontinuous water-water emulsions, are highly unstable and tend to separate very quickly into equilibrium phases, unless an effective stabilization is achieved.

Some authors have used microfluidic techniques for the preparation of such $\mathrm{W} / \mathrm{W} / \mathrm{W}$ multiple emulsions, achieving a good control over the structure and morphology of multiple droplets. Microfluidics are certainly useful techniques for preparation of multiple droplets with excellent monodispersity. For example, nicely controlled multiple W/W/W emulsions can be achieved by preparing $\mathrm{W} / \mathrm{W}$ emulsions by microfluidics and then inducing phase separation inside these droplets $^{52}$. Moreover, microfluidic techniques have also been used to determine equilibrium concentrations and drawing phase diagrams, determining the binodal phase boundary ${ }^{54}$.

However, microfluidic techniques are not easily scalable at low cost, and large production volumes cannot be achieved. To our knowledge, a simple and cost-effective method for the production at large scale of multiple W/W/W emulsions, has not been reported. In the present work, our aim was the formation of multiple water-in-water-in-water emulsions by methods based on phase transitions and nucleation and growth phenomena. The present work demonstrates that simple physical chemical principles can be applied to spontaneously form multiple W/W/W emulsions. Moreover, it should be inferred that these novel structured aqueous emulsions might have much technological interest in formulation of oil-free and surfactant-free systems.

Another important objective is the preparation of multiple $\mathrm{W} / \mathrm{W} / \mathrm{W}$ emulsions using nontoxic and edible components. In the present work, we have focused our attention in the gelatinmaltodextrin system, since both polymers are biocompatible and information about its phase behaviour is well known ${ }^{53,55,56}$. Moreover, the interactions between these molecules can be switched from attractive to repulsive. Considering that the isoelectric point of gelatin is $\mathrm{pH}=4.5$, depending on $\mathrm{pH}$ conditions it has a net-positive or net-negative charge. In addition, gelatin solutions allow the formation of physical gels at temperatures below its gelation point. Therefore, systems derived from gelatin-maltodextrin aqueous mixtures might be useful for the preparation of structured microgels. For all these reasons, the present work aims to obtain multiple water-in-water-in-water (W/W/W) emulsions in gelatin-maltodextrin aqueous mixtures, by a simple and cost-effective method. 


\section{Products and Methods}

\section{Products}

Gelatin (herein abbreviated as G) was bovine skin type B, with gel strength $\sim 225 \mathrm{~g}$ Bloom and a molecular mass around 50-100 kDa. Maltodextrin (abbreviated as M) had a dextrose equivalent of 4.0-7.0 and a molecular mass of $3.6 \mathrm{kDa}$. These two main components were both supplied by Sigma-Aldrich (Spain).

Fluorescein 5(6)-isothiocyanate (abbreviated as FITC) was purchased from Sigma-Aldrich with a purity $\geq 90 \%$. The labeling of maltodextrin with FITC was improved with the addition of anhydrous pyridine, purity $\geq 99.8 \%$, and dibutyltin dilaureate $\left(\mathrm{C}_{32} \mathrm{H}_{64} \mathrm{O}_{4} \mathrm{Sn}\right)$, purity $\geq 95 \%$, both supplied by Sigma-Aldrich. Rhodamine B isothiocyanate (denoted as RBITC) was also purchased from Sigma-Aldrich, with a mixture of isomers and unknown purity.

Mucin from porcine stomach, Type III, bound sialic acid 0.5-1.5 \% (partially purified powder), was supplied by Sigma-Aldrich. For some additional experiments, type II mucin was also used, with bound sialic acid $\leq 1.2 \%$. This mucin sample was also provided by Sigma-Aldrich.

The solvents were ethanol 95\%, supplied by Alcoholes Montplet and dimethyl sulfoxide (DMSO), with a purity $\geq 99.9 \%$, supplied by Merck.

Other products were $\mathrm{NaOH}$, in form of pellets ( $>97 \%)$, supplied by Carlo Erba, and acetic acid glacial, pure pharma grade, supplied by Panreac. Water had always been deionized and filtered with a Milli-Q ${ }^{\circledR}$ Millipore Synergy Smart UV system, ensuring a water quality with a minimum of $18 \mathrm{M} \Omega \cdot \mathrm{cm}$ resistivity.

\section{Methods}

Preparation of stock solutions

Gelatin stock solutions, typically at $25 \mathrm{wt} \%$ concentration, were prepared by dissolving gelatin in hot water at $60^{\circ} \mathrm{C}$, using a hotplate water bath with magnetic stirring at 500rpm, during approximately $30 \mathrm{~min}$. Maltodextrin stock solutions were usually prepared at $30 \mathrm{wt} \%$ concentration. These solutions were prepared by heating at $95^{\circ} \mathrm{C}$, with magnetic stirring at 500 rpm until complete dissolution, which also required approximately $30 \mathrm{~min}$.

These stock solutions were used for a period of several days. At room temperature, gelatin concentrated solutions formed gels and maltodextrin solutions precipitate. Consequently, both stock solutions were reheated briefly at $60^{\circ} \mathrm{C}$, until forming homogeneous solutions, just before using them.

The stock dispersions of mucin were prepared just before their use, considering that mucin is insoluble in water, but it can be dispersed as a particle suspension. Accordingly, samples were stirred at $45^{\circ} \mathrm{C}$ during $30 \mathrm{~min}$ and afterwards were treated by ultrasonication, with a Sonopuls MS72 Titanium probe, from Bandelin using a Bandelin Sonopuls ultrasonic homogenizer HD3200 instrument. The ultrasonication treatment consisted in applying 5 second on $/ 5$ second 
off cycles during a total of $60 \mathrm{~s}$, using a $40 \%$ amplitude. Preliminary experiments demonstrated that this treatment allows obtaining particle suspensions with a slow sedimentation rate. These suspensions were used for short periods of time, and always were stirred again by hand before mixing it with maltodextrin.

\section{Labelling with fluorescent dyes}

Fluorescence labelling was required for proper observations by optical microscopy, and depending of the case, either gelatin or maltodextrin were covalently labelled with FITC, which produces fluorescent emissions with green colour. Gelatin was labelled by preparing a solution of $2 \mathrm{wt} \%$ FITC, dissolved in DMSO. This solution was added to gelatin stock solutions, at 1:1000 mixing ratio, and these mixtures were stirred for a minimum of $2 \mathrm{~h}$ at $60^{\circ} \mathrm{C}$. It was believed that the residual amount of DMSO did not modify phase behaviour, and thus, labelled gelatin was not purified. Mucin was labelled with exactly the same procedure, but using RBITC instead of FITC. RBITC imparted a red colour to mucin, when observed by fluorescence optical microscopy.

In the case of labelling maltodextrin, which is less reactive than gelatin, the method described by De Belder and Granath ${ }^{57}$ was adapted. In a typical example, 10 grams of maltodextrin were dispersed in $100 \mathrm{~mL}$ anhydrous DMSO, which contained a few drops of pyridine. Then, $25 \mathrm{mg}$ of FITC were added, followed by the addition of $200 \mathrm{mg}$ dibutylin dilaureate. The mixture was kept at $65^{\circ} \mathrm{C}$, for 2 hours under gentle stirring. Afterwards, FITC-labeled maltodextrin was precipitated by adding ethanol $(95 \% \mathrm{v} / \mathrm{v})$. The filtered precipitated was washed several times with ethanol, to remove the unreacted free dye. Finally, the FITC-labelled maltodextrin was dried in a desiccator, in presence of phosphorus pentoxide, or alternatively, it could be dried in an oven at mild conditions $\left(45^{\circ} \mathrm{C}\right)$.

\section{Study of Phase Behaviour}

Mixtures of gelatin and maltodextrin of different concentrations were prepared in order to study their phase behaviour in water. Samples were introduced inside $5 \mathrm{~mL}$ vials by mixing selected amounts of gelatin and maltodextrin stock solutions, and then adding purified water to reach the final concentrations. Mixtures were always manipulated at approximately $50^{\circ} \mathrm{C}$, to prevent both gelatin gelation and maltodextrin precipitation.

Final concentrations of gelatin were between $0-12 \mathrm{wt} \%$, and concentrations of maltodextrin were in the range between $0-22 \mathrm{wt} \%$. Afterwards, vials were closed, vortex agitation was applied during approximately 10 seconds, and vials were introduced into a thermostated bath, keeping temperature constant at $50{ }^{\circ} \mathrm{C}$. The possible presence of segregated equilibrium phases was observed by naked eye, during a minimum period of 5 days. This time for equilibration was selected because preliminary experiments showed that phase separation was relatively slow; and a certain time was required to observe changes in sample aspect by naked eye. Observations were not extended for much longer periods of time, to prevent the possibility of slow thermal hydrolysis of gelatin. 
Water-in-water emulsions, which consisted of gelatin-maltodextrin mixtures, were prepared with selected compositions by two different methods: phase transition and mechanical agitation. Independently of the emulsification method used, all the emulsions were prepared and kept at a constant temperature of $50^{\circ} \mathrm{C}$. The phase transition method consisted on the mixture of components at $\mathrm{pH} \approx 3-4$, followed by a fast increase of $\mathrm{pH}$ to $\approx 5$, mixing all the system at 500 $\mathrm{rpm}$ for $5 \mathrm{~min}$. When mechanical agitation was used, the mixture gelatin-maltodextrin at $\mathrm{pH} \approx 5$ is mixed by Ultraturrax at $8000 \mathrm{rpm}$ for $2 \mathrm{~min}$.

\section{Determination of gel point by rheological measurements}

The gel temperature of gelatin (data shown as Supporting Information, Fig. S4) was determined by measuring viscosity as a function of temperature, from 50 to $15{ }^{\circ} \mathrm{C}$, cooling down at 2 ${ }^{\circ} \mathrm{C} / \mathrm{min}$. The rheometer was an AR-G2 from TA Instruments, with a cone-plate geometry of $40^{\circ}$ and $105 \mu \mathrm{m}$ of gap.

\section{Ultrasonication}

Mucin particles were dispersed in water by ultrasonication, using a Sonoplus Ultrasonic Homogenizer HD3200, manufactured by Bandelin. It was operated at $40 \%$ amplitude for 1 minute, using $5 \mathrm{~s}$ on $/ 5 \mathrm{~s}$ off cycles.

\section{Particle sizing}

Particle size distribution of mucin particle suspensions was obtained by Light Diffraction, using a MasterSizer 2000 Malvern instrument, equipped with a liquid dispersing unit. The average particle size was calculated as the de Brouckere mean diameter, $\mathrm{D}[4,3]$, and polydispersity was quantified as $\mathrm{D}(\mathrm{v} 0.5) /(\mathrm{D}(\mathrm{v} 0.9)-\mathrm{D}(\mathrm{v} 0.1))$, denoted as Span, where $\mathrm{D}(\mathrm{v} 0.1), \mathrm{D}(\mathrm{v} 0.5)$ and $\mathrm{D}(\mathrm{v} 0.9)$ are the percentiles in volume distribution.

\section{Observations by Laser Scanning Confocal Microscopy (LSCM).}

The microscope was a Leica TCS SP5 laser scanning confocal microscope (Leica Microsystems Heidelberg GmbH, Manheim, Germany) equipped with a DMI6000 inverted microscope, blue diode $(405 \mathrm{~nm})$, Argon (458/476/488/496/514), diode pumped solid state $(561 \mathrm{~nm})$ and $\mathrm{HeNe}$ (594/633nm) lasers and HCX PLAN APO 63x Glycerol (Numerical Aperture 1.3, 280 microns working distance) immersion objective lens.

FITC and RBITC images were acquired sequentially using 488 and 561 laser lines, AOBS (Acoustic Optical Beam Splitter) as beam splitter, emission detection ranges at 500-550 nm and 571-625 nm, respectively, and setting the confocal pinhole at 1 Airy units. Simultaneously, transmitted light was also acquired. Images were acquired at $400 \mathrm{~Hz}$ in a 1024 x 1024 pixels format, line average 4 . For 3D reconstruction, z sectioning was performed every $200 \mathrm{~nm}$. 


\section{Results and Discussion}

\section{Phase Behaviour}

Phase diagrams were studied as a function of gelatin and maltodextrin concentrations, at $50{ }^{\circ} \mathrm{C}$ constant temperature. This temperature was selected because solubility of maltodextrin is much higher at $50{ }^{\circ} \mathrm{C}$ than at room temperature, and also because this temperature is significantly higher than the gelation point of gelatin $\left(\approx 17.5^{\circ} \mathrm{C}\right.$, shown in Fig. S4 as Supporting Information), which accelerates phase separation without affecting its chemical stability. The $\mathrm{pH}$ of gelatinmaltodextrin mixtures $(\mathrm{pH} \approx 5)$ was not buffered. The phase diagram is shown in Fig. 1a.

Three different regions can be observed in the phase diagram, depending on composition. At low gelatin and maltodextrin concentrations, one single phase (L) is formed, demonstrating that gelatin and maltodextrin are miscible at low concentrations. However, at higher concentrations of both molecules, a solid phase (S) is observed, coexisting with the liquid phase, as a precipitate $(\mathrm{L}+\mathrm{S})$. Increasing even more concentrations of either gelatin or maltodextrin, the liquid phase splits into two equilibrium liquid phases, which are in equilibrium with the solid phase $\left(\mathrm{L}_{1}+\mathrm{L}_{2}+\mathrm{S}\right)$. An illustrative example of this coexistence of two liquid phases and a solid precipitate is shown in the Supporting Information (Fig. S1). This split occurs on the binodal line, which is the phase boundary between one liquid phase and the coexistence of two segregated liquid phases. Overall, our phase diagram (Fig. 1a) is quite similar to those reported in other studies of gelatin-maltodextrin aqueous mixtures ${ }^{55,56,58,59}$. Small differences can be attributed to different origins and purities of the macromolecules. In a recent publication, it has been shown that the phase behaviour of gelatin/maltodextrin mixtures is determined by intermolecular interactions, described in terms of Flory-Huggins parameters ${ }^{59}$, and thus it can be very sensitive to small amounts of impurities.

The phase diagram under slightly acidic conditions, by adding a constant concentration of 3.3 wt $\%$ acetic acid, is shown in Fig. $1 b$. Reduction in $\mathrm{pH}$, from $\approx 5$ to $\approx 3.5$, produces a great change in phase behaviour. The two equilibrium liquid phases $\left(\mathrm{L}_{1}+\mathrm{L}_{2}\right)$ present at $\mathrm{pH} \approx 5$ (Figure 1a) merge on one single liquid phase at $\mathrm{pH} \approx 3.5$ (Fig. 1b). The isoelectric point of gelatin polypeptide chains is approximately $\mathrm{pI}=4.5$, and therefore, gelatin is negatively charged at $\mathrm{pH}>4.5$, whereas is has a net positive charge below this $\mathrm{pH}$. It is known that liquid-liquid separation in gelatin-maltodextrin aqueous mixtures is promoted by conformational ordering and aggregation of gelatin chains ${ }^{55,58}$, and it seems evident that such phase separation does not occur in acidic conditions. 
(a) $\mathrm{pH} \approx 5$

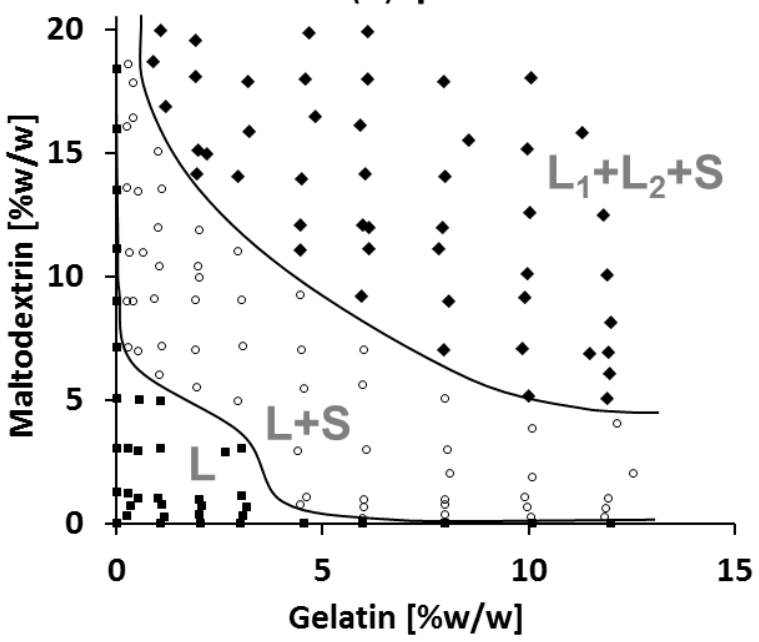

(b) 3.3 wt\% Acetic Acid (pH 3-4)

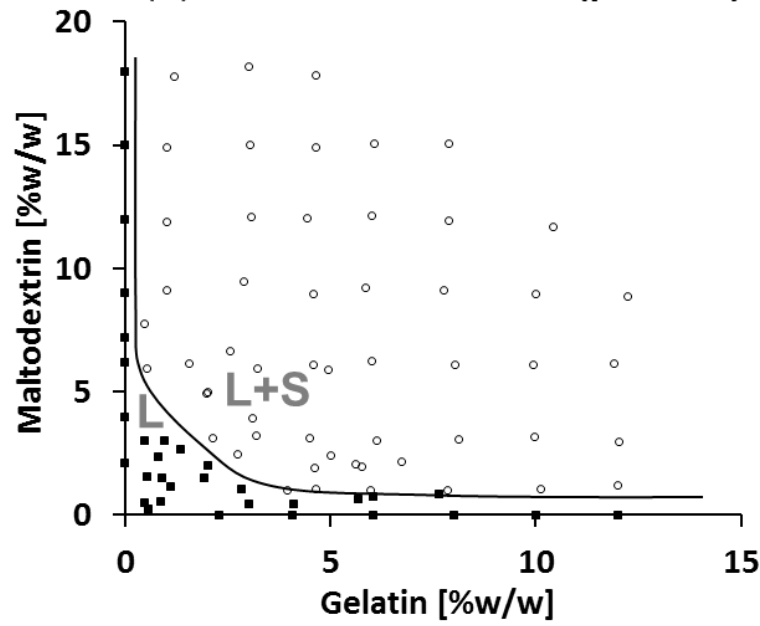

Fig. 1. Phase diagrams of pseudoternary systems composed of water, gelatin and maltodextrin, as a function of maltodextrin and gelatin concentrations, at $50{ }^{\circ} \mathrm{C}$ constant temperature during 5 days: (a) phase diagram at $\mathrm{pH} \approx 5$, in absence of any $\mathrm{pH}$ modifier, and (b) Phase diagram in presence of acetic acid at $3.3 \mathrm{wt} \%$ constant concentration, which induced $\mathrm{pH}$ values between 3 and 4 , depending on the acetic acid / gelatin ratio. The different regions are shown, indicating one homogeneous phase (L); a liquid phase in presence of a solid precipitate $(L+S)$; and two equilibrium liquid phases coexisting with a solid phase $\left(L_{1}+L_{2}+S\right)$. (The Phase diagram shown in (b) has been redrawn from a previous version ${ }^{59}$, with copyright permission).

The formation of the solid phase (S) occurs very slowly. Experimentally, precipitation of the solid is not observed by optical microscopy in freshly prepared samples, and its detection requires several days of observations. For this reason, phase diagrams shown in Fig. 1 were obtained by equilibrating samples at constant temperature during 5 days. Because of such slow formation, the existence of this solid phase can be neglected at short periods of time, and aqueous mixtures within the $\mathrm{L}+\mathrm{S}$ region can be treated as a single pseudophase.

It has been reported that this $\mathrm{S}$ phase is mainly composed of maltodextrin and its precipitation was attributed to changes in molecular conformation ${ }^{55}$. In any case, phase behaviour of gelatinmaltodextrin aqueous mixtures is rather complex, and this study is not in the scope of the present paper. Detailed studies of phase behaviour in gelatin/maltodextrin mixtures can be found elsewhere ${ }^{46,55,59,60}$. In a recent work ${ }^{59}$, it has been confirmed by Raman spectroscopy that the $\mathrm{S}$ precipitate is mainly composed of maltodextrin, but its composition depends on the overall composition of the mixture, as inferred from simulations of Flory-Huggins interaction parameters.

A very interesting aspect is the dependence on $\mathrm{pH}$. Compositions above the binodal line at $\mathrm{pH}$ $\approx 5$, thus forming two liquid phases, appear with only one liquid phase at $\mathrm{pH} \approx 3.5$. Then, one could form W/W emulsions by changing $\mathrm{pH}$. Maltodextrin-in-gelatin $(\mathrm{M} / \mathrm{G})$ emulsions can be formed by generating a phase transition, from one liquid phase, $L$, to two liquid phases, $L_{1}+L_{2}$. This phase transition can be induced by increasing $\mathrm{pH}$ from 3.5 to 5 . Results are shown in the next section. 


\section{Formation of water-in-water emulsions by $\mathrm{pH}$-induced phase transition}

Various compositions, with different gelatin/maltodextrin weight ratios ranging from 4/20 to $12 / 5$, were prepared. Temperature was kept constant at $50{ }^{\circ} \mathrm{C}$ by placing the sample vials in thermostated water baths. All compositions were within the $\mathrm{L}+\mathrm{S}$ region at $\mathrm{pH} \approx 3.5$, and within the $\mathrm{L}_{1}+\mathrm{L}_{2}+\mathrm{S}$ region of the phase diagram at $\mathrm{pH} \approx 5$. Samples were prepared at acidic $\mathrm{pH}$ with 3.3 $\mathrm{wt} \%$ acetic acid, and $\mathrm{pH}$ was increased by addition of small volumes of $\mathrm{NaOH}$ in aqueous solution, reaching $\mathrm{pH} \approx 5$. The final emulsions were observed by fluorescence optical microscopy, aided by labelling of maltodextrin with FITC. Illustrative examples of different emulsion types are shown in Fig. 2. The solid precipitate, S, was never observed, since its formation would require longer periods of time.

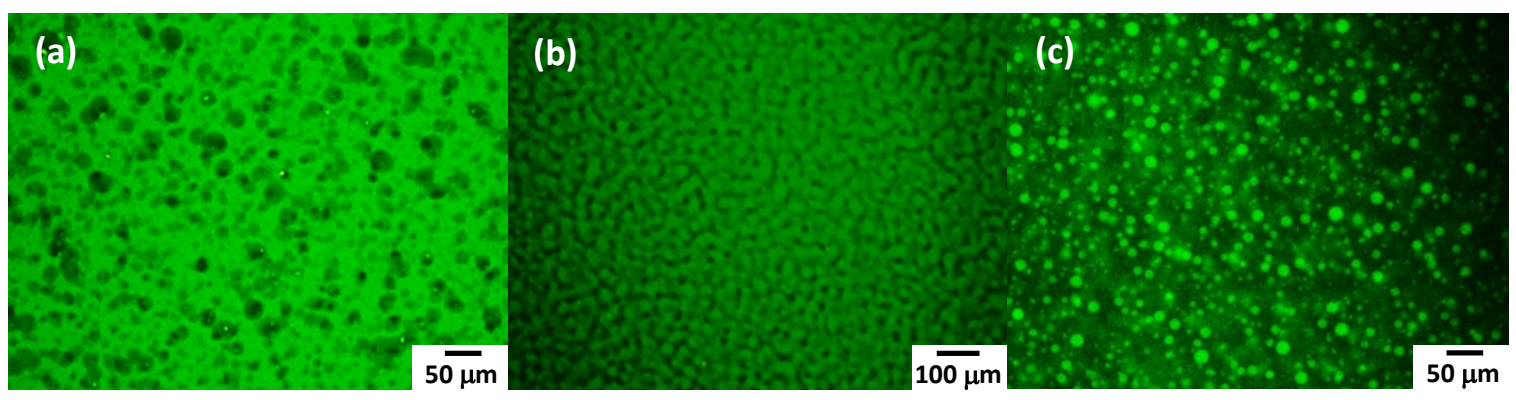

Fig. 2. Images captured by fluorescence optical microscopy, in gelatin-maltodextrin aqueous emulsions, prepared by addition of $\mathrm{NaOH}$, reaching $\mathrm{pH} \approx 5$. The green regions correspond to domain enriched in maltodextrin labelled with FITC, and the dark regions indicate domains that contain mainly gelatin. (a) gelatin-in-maltodextrin (G/M) emulsion, prepared at 4 wt\% gelatin and $20 \mathrm{wt} \%$ maltodextrin; (b) Unstable bicontinuous emulsion with 5 wt\% gelatin and 12 wt\% maltodextrin; and (c) maltodextrin-ingelatin (M/G) emulsion that contains $12 \mathrm{wt} \%$ gelatin and $5 \mathrm{wt} \%$ maltodextrin.

Fig. 2 shows examples of emulsions formed by increasing pH. Emulsion droplets formed immediately after adding $\mathrm{NaOH}$, in all compositions above the binodal line of the phase diagram, as expected. The composition with a larger volume of the maltodextrin phase ( $4 \mathrm{wt} \%$ gelatin, $20 \mathrm{wt} \%$ maltodextrin, in Fig. 2a) produced gelatin-in-maltodextrin droplets, whereas the composition with higher volume of gelatin phase ( $12 \mathrm{wt} \%$ gelatin, $5 \mathrm{wt} \%$ maltodextrin, Fig. $2 \mathrm{c}$ ) induced the formation of maltodextrin-in-gelatin emulsions.

Moreover, the transient formation of bicontinuous emulsions, at $5 \mathrm{wt} \%$ gelatin and $12 \mathrm{wt} \%$ maltodextrin (Fig. 2b), was an interesting and unexpected result. It was observed that this emulsion formed rather quickly, but it was highly unstable and rapidly coarsened by coalescence. Therefore, it could only be observed within a short period of time under the optical microscope. This bicontinuous emulsion presents a high resemblance to other bicontinuous morphologies obtained by spinodal decomposition ${ }^{61}$. The phase diagram, Fig. 1a, shows that its composition is close to the binodal line in the centre of the diagram. Therefore, one can presume that it probably lies inside the spinodal decomposition region of the phase diagram. In any case, it is remarkable that spinodal decomposition, usually observed by temperature quenching in oilwater mixed systems, it can also be produced in water-in-water emulsion systems prepared using water-soluble biocompatible polymers, in which $\mathrm{pH}$ induced homogeneous phase separation. 
3. Comparison of $M / G$ emulsions formed by phase transition and emulsions prepared by a conventional agitation method

The present work has demonstrated that $\mathrm{W} / \mathrm{W}$ emulsions can be formed by a phase transition method. The resulting emulsions can be compared to those emulsions prepared by using a conventional method based on applying high shear agitation. This was done by checking the stability of the emulsions after a period of five days.

It is well-known ${ }^{1}$ that $\mathrm{W} / \mathrm{W}$ emulsions can be prepared by applying agitation in aqueous biphasic systems. In this context, W/W emulsions are governed by the same principles as $\mathrm{W} / \mathrm{O}$ or $\mathrm{O} / \mathrm{W}$ emulsions and could show the same instability processes. First of all, the stability of these emulsions was compared by visual observation as a function of time, evaluating the influence of the emulsification method. Fig. 3 shows the aspect of emulsions, kept at $50{ }^{\circ} \mathrm{C}$ for 5 days. The bottom layer of these samples is mainly a sedimented maltodextrin-rich solution, which is more dense, and the upper layer corresponds to the gelatin-rich residual emulsion. It clearly illustrates the influence of the emulsification process. Emulsions A and B, prepared by phase transition, showed a homogenous aspect without visible phase separation after 5 days. In contrast, the interface between maltodextrin and gelatin could be observed in emulsions $\mathrm{C}$ and $\mathrm{D}$ prepared by agitation, at $\mathrm{t}=3 \mathrm{~h}$, indicating that phase separation already occurred in emulsions prepared by this method. By extending time to 5 days at $50^{\circ} \mathrm{C}$, samples $\mathrm{A}$ and $\mathrm{B}$ only showed a slight increase of turbidity without significant sedimentation. However, emulsions C and D showed sedimentation of denser maltodextrin droplets after 5 days, and a clear layer of maltodextrin separated phase was observed in these samples. These results certainly reflect the higher stability of emulsions prepared by phase transition (samples A and B), which was induced by increasing $\mathrm{pH}$. In conclusion, the preparation method greatly influences emulsion stability.
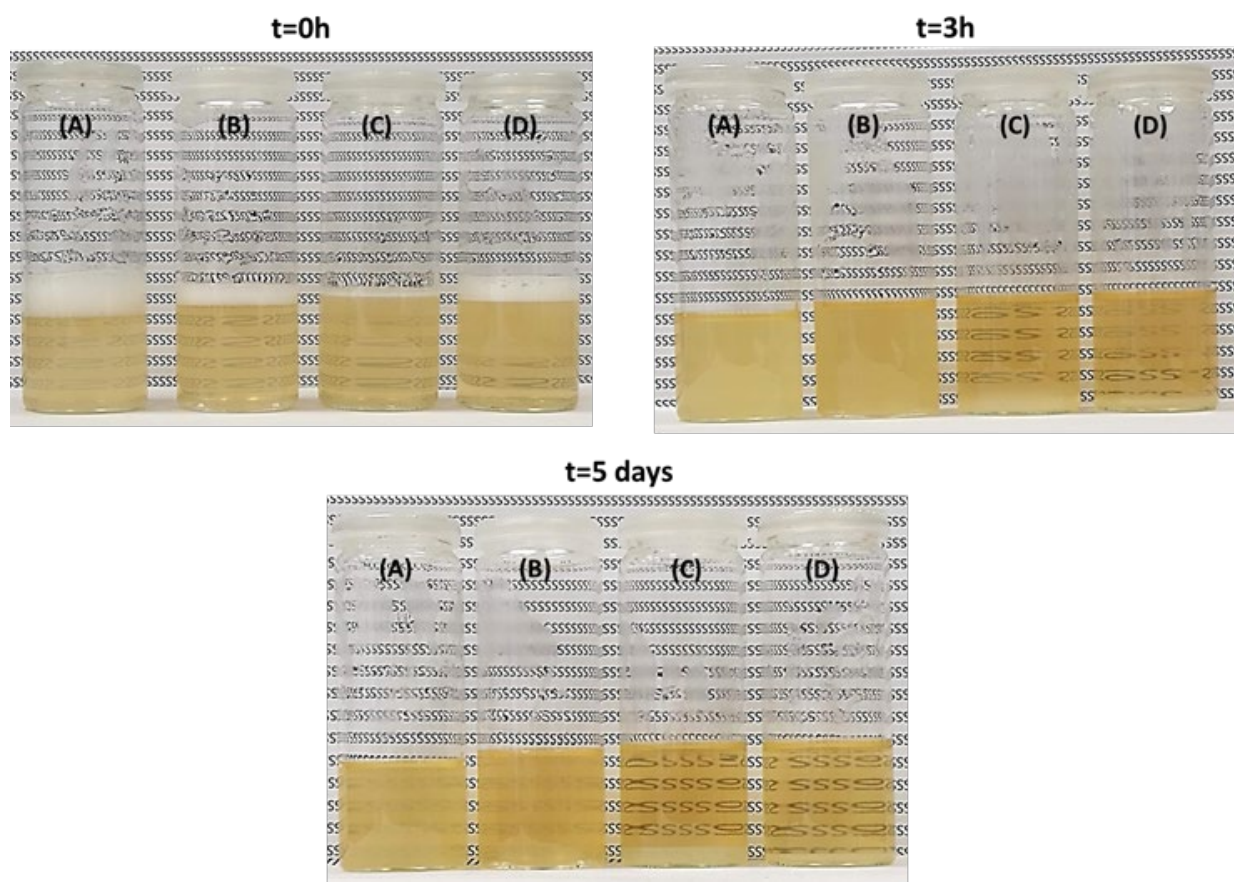
Fig. 3. Comparison of maltodextrin-in-gelatin emulsions prepared by phase transition ( $A$ and $B$ ) and prepared by agitation ( $C$ and $D$ ), observed during 5 days. (A): 11 wt\% gelatin and 6.4 wt\% maltodextrin; (B): 12 wt\% gelatin and 5 wt\% maltodextrin; (C) 12 wt\% gelatin and 7 wt\% maltodextrin; (D): 12 wt\% gelatin and $5 \mathrm{wt} \%$ maltodextrin.

Small volumes of residual emulsions, located near the interfaces, were extracted with syringes and were observed by fluorescence optical microscopy, using maltodextrin labelled with FITC. Colloidal stability of emulsions was qualitatively evaluated by comparing droplet size and polydispersity at $t \approx 3$ hours and $t \approx 5$ days (Fig. 4).

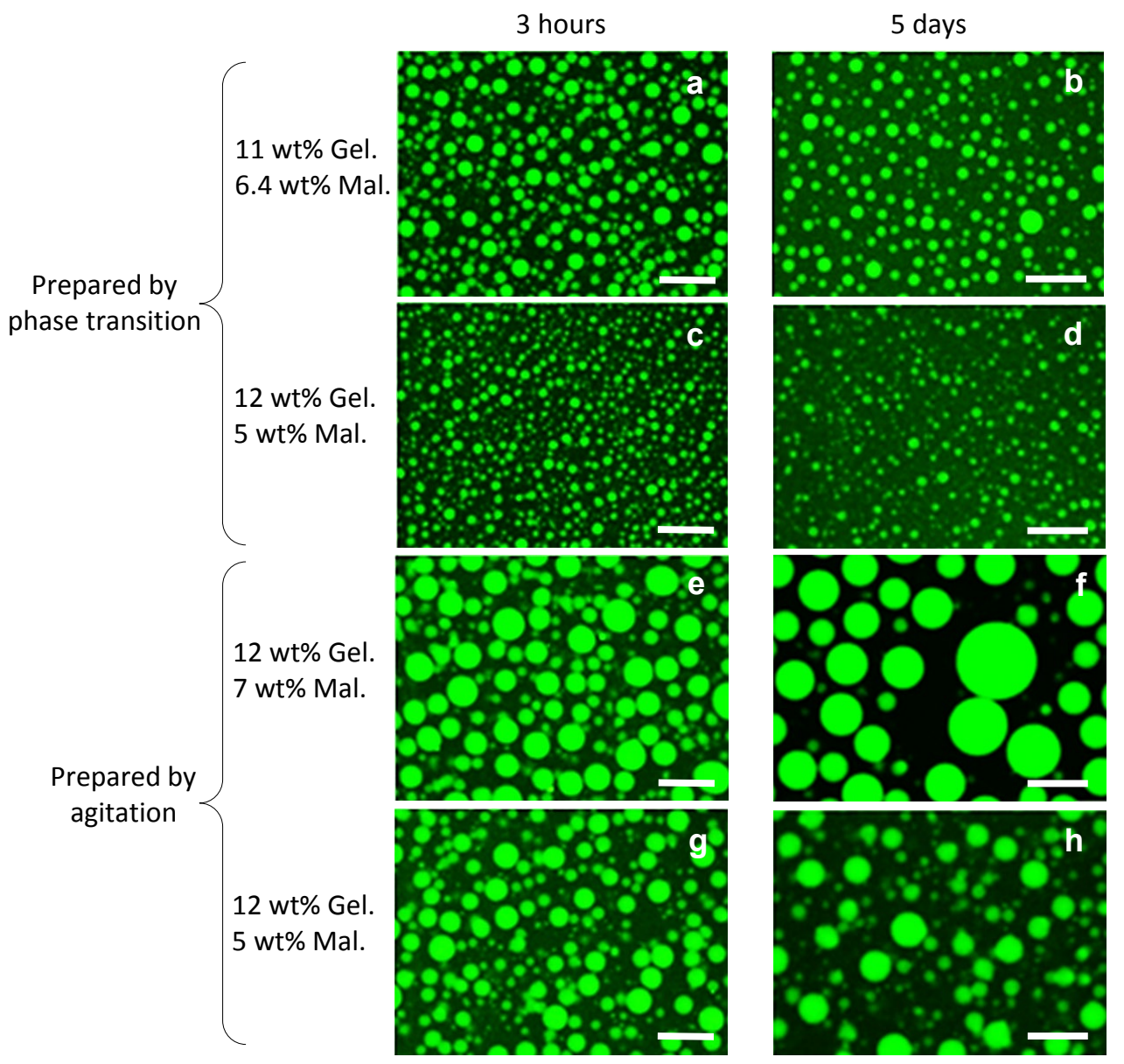

Fig. 4. Fluorescence optical micrographs of maltodextrin-in-gelatin $(M / G)$ emulsions at $t=3$ hours and $t=5$ days. Maltodextrin was labelled with FITC, marked in green color. Samples with 11 wt\% gelatin and 6.4 wt\% maltodextrin ( $a$ and $b$ ) and with 12 wt\% gelatin and 5 wt\% maltodextrin (c and d), were prepared by phase transition. Samples with 12 wt\% gelatin and 7 wt\% maltodextrin (e and f) and with 12 wt\% gelatin and 5 wt\% maltodextrin ( $g$ and $\mathrm{h}$ ) were all prepared by mechanical agitation. The white scale bars correspond to $50 \mu \mathrm{m}$.

The influence of the preparation method in the properties of water-in-water emulsions is clearly shown in the micrographs of Fig. 4. Phase transition method allows obtaining emulsions with smaller droplet size and lower polydispersity (Fig. 4a-d) than using the mechanical agitation method (Fig. 4e-h). The transition method based on a $\mathrm{pH}$ change together with mild agitation gave rise to emulsions with droplet size between 5-10 $\mu \mathrm{m}$ (Fig. 4a and c), depending on composition. This means a significant decrease in droplet size, in comparison to mechanical 
agitation method, which produces droplet sizes from 20 to $40 \mu \mathrm{m}$ at initial time (Fig. $4 \mathrm{e}$ and g). Indeed, in addition to these results, the characterization of theses emulsions after 5 days reflects clearly the improvement of emulsion stability by using the phase transition method, obtaining lower variation of droplet size (Fig. $4 \mathrm{~b}$ and d). Emulsions prepared by mechanical agitation after 5 days (Fig. $4 \mathrm{f}$ and $\mathrm{h}$ ) tend to present a coalescence process followed by sedimentation of the dispersed phase (as observed in Fig. 3). Therefore, Fig. 4f and 4h clearly show an increase of droplet size as a function of time, in comparison with Fig. $4 \mathrm{e}$ and $\mathrm{g}(\mathrm{t}=3$ hours $)$.

These results confirm that emulsions with smaller droplet size and improved stability can be prepared by phase transition. Interestingly, one could infer that smaller sizes are produced because this method constitutes a bottom-up approach where droplets are formed by condensation, instead of a top-down emulsification by mechanical dispersion. Moreover, it should be remarked that a relatively small droplet size was obtained without any surfactant/particle to stabilize them.

Since the formation of a W/W emulsion with a composition of $12 \% \mathrm{G}$ and $5 \% \mathrm{M}$ by phase transition method formed the smallest droplet size, this composition was chosen to be further continued with for the formation of multiple emulsions.

4. Formation of multiple (double) maltodextrin-in-gelatin-in-maltodextrin $(M / G / M)$ emulsions by addition of $M / G$ emulsions to maltodextrin solutions

Conventional oil-in-water-in-oil $(\mathrm{O} / \mathrm{W} / \mathrm{O})$ or water-in-oil-in-water $(\mathrm{W} / \mathrm{O} / \mathrm{W})$ double emulsions are usually prepared by two-step emulsification methods, in which single emulsions, either $\mathrm{O} / \mathrm{W}$ or $\mathrm{W} / \mathrm{O}$, are introduced in an external phase, oily or aqueous, respectively.

In the present work, double water-in-water-in-water (W/W/W) emulsions were prepared using a similar two-step approach, but using two aqueous immiscible phases in absence of oil and surfactants. The first step was to prepare a primary emulsion (PE) of maltodextrin-in-gelatin $(\mathrm{M} / \mathrm{G})$ type, with composition $5 \mathrm{wt} \%$ maltodextrin and $12 \mathrm{wt} \%$ gelatin, by using the method described in the previous section, inducing a phase transition that produces a splitting of the liquid phase into two segregated liquids by increasing $\mathrm{pH}$. The second step was the dropwise addition of the PE, during 3 minutes, to a maltodextrin solution under agitation with a magnetic stirrer. Multiple (double) emulsions were identified by fluorescence optical microscopy, by labelling maltodextrin with FITC. Fig. 5 shows illustrative examples of multiple maltodextrinin-gelatin-in-maltodextrin (M/G/M) emulsions, obtained with $60 \mathrm{wt} \%$ of M/G primary emulsion and $40 \mathrm{wt} \%$ of maltodextrin as external phase.
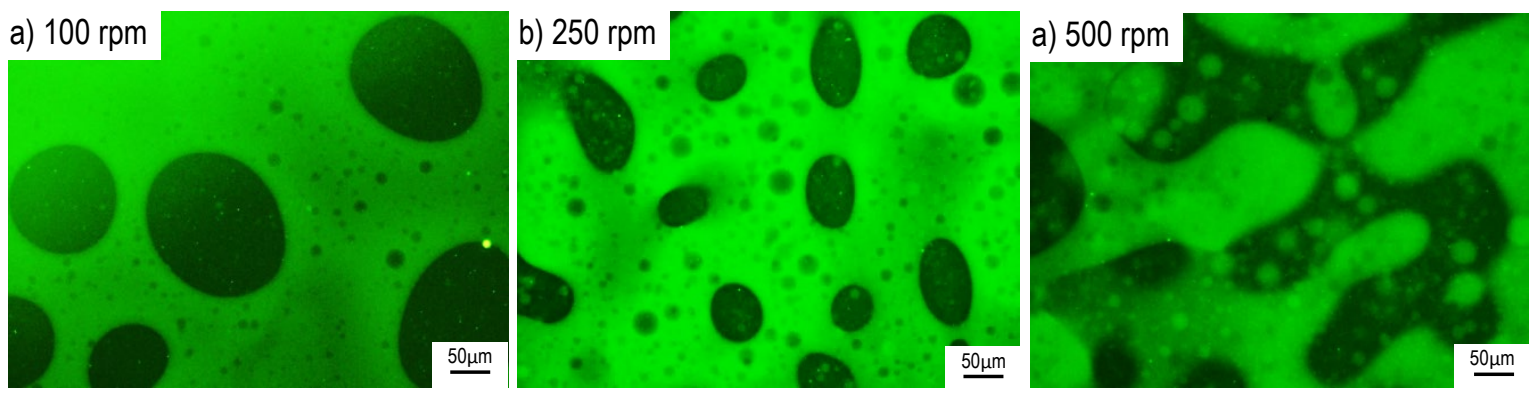
Fig. 5. Maltodextrin-in-gelatin-in-maltodextrin (M/G/M) multiple emulsions, prepared at different stirring conditions. The composition of primary emulsion (PE) was $12 \mathrm{wt} \% \mathrm{G}$ and $5 \mathrm{wt} \% \mathrm{M}$. PE was added to a $30 \mathrm{wt} \% \mathrm{M}$ solution, at 60/40 PE/M weight ration. In all cases, maltodextrin was labelled with FITC, and the images were taken just after the preparation of the sample. (a) Addition of $M / G$ to $M$ at 100 rpm; (b) applying $250 \mathrm{rpm}$; and (c) at $500 \mathrm{rpm}$.

Slow agitation (100 rpm, Fig. 5a) produces emulsions with large M/G/M double droplets (diameter $\approx 200 \mu \mathrm{m}$ ), which contain inner small droplets of maltodextrin. However, droplets are not homogenous, since presence of small simple G/M emulsion droplets is observed (dark small droplets seen in Fig. 5a and 5b). Agitation at $250 \mathrm{rpm}$ (Fig. 5b) reduces the size of double droplets, down to approx. $40 \mu \mathrm{m}$, but the number of single droplets is increased. Even faster agitation breaks primary emulsion droplets and multiple emulsions are not clearly formed (Fig. $5 c)$.

The stability of the emulsions shown in Fig. $5 \mathrm{a}$ and $5 \mathrm{~b}$ was qualitatively assessed by visual inspection. These samples showed fast phase separation, after 3 hours at $37^{\circ} \mathrm{C}$ (Fig. S2, in Supporting Information). Therefore, the above multiple emulsions were highly polydisperse and very unstable, concluding that a better method for emulsification had to be searched, and stability of multiple emulsion droplets required much improvement. In next section, the formation of multiple $\mathrm{W} / \mathrm{W} / \mathrm{W}$ emulsions by a straightforward method, based on another phase transition, is reported.

5. Formation of multiple (double) maltodextrin-in-gelatin-in-maltodextrin $(M / G / M)$ emulsions by decreasing temperature of $M / G$ emulsions

It is known that solubility of maltodextrin, in the gelatin phase, decreases at low temperatures, and this temperature-dependence can be used to form maltodextrin-in-gelatin emulsions ${ }^{58,62}$, by a temperature quench of homogeneous mixtures. Our previous studies ${ }^{46}$ have indicated that it was possible to obtain multiple emulsions by changing the temperature in mixtures maltodextrin-gelatin with compositions close to the binodal line. From these initial results, phase behaviour was studied at different temperatures above the gelation temperature of gelatin. The results indicated that the binodal line is slightly shifted to higher maltodextrin concentrations when increasing temperature (Fig. 6). This confirms that solubility of maltodextrin, inside gelatin aqueous droplets, decreases with temperature. The interesting consequence is that gelatin phase could become oversaturated with maltodextrin, simply by decreasing temperature. Then, maltodextrin droplets can form spontaneously by condensation from this oversaturated composition. 


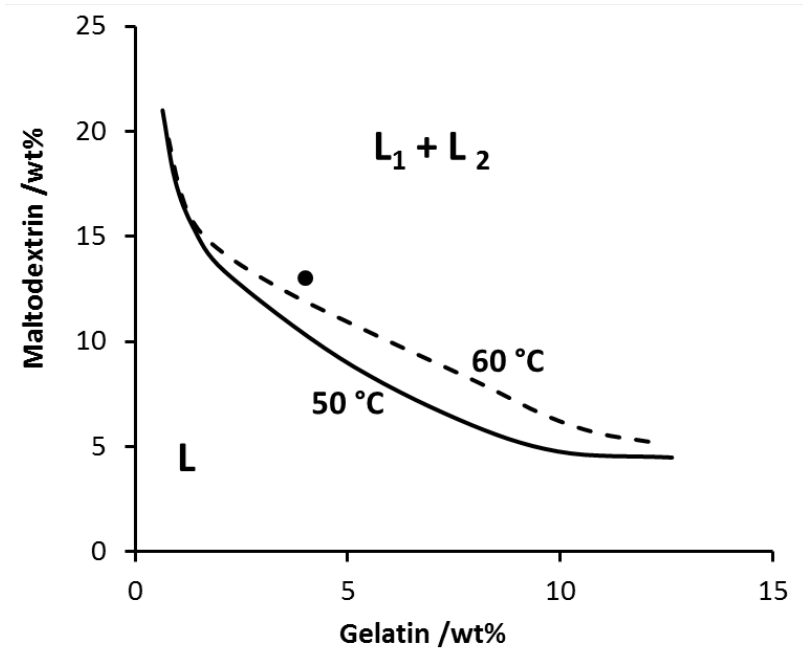

Fig. 6. Binodal lines, for two different temperatures $\left(50\right.$ and $\left.60^{\circ} \mathrm{C}\right)$, as the boundary between the region with one liquid phase $(L)$ and the region with two aqueous liquid phases $\left(L_{1}+L_{2}\right)$. The presence of a third phase (solid-like S phase) was not studied. The black circle indicates the 4 wt\% gelatin / 13 wt\% maltodextrin composition, which is used below.

Gelatin-in-maltodextrin $(\mathrm{G} / \mathrm{M})$ emulsions, in a composition of $4 \mathrm{wt} \%$ gelatin and $13 \mathrm{wt} \%$ maltodextrin, were prepared at $50{ }^{\circ} \mathrm{C}$ by mechanical agitation, and secondary phase separation occurred by cooling at approximately $2{ }^{\circ} \mathrm{C} / \mathrm{min}$ velocity, controlling the temperature of the hot stage. Droplet evolution was observed by optical microscopy aided with differential interference contrast, enhancing low contrast due to the similarity of refractive indexes of both aqueous phases (images shown in Fig. 7). The spontaneous formation of maltodextrin inner droplets was observed at approximately $30{ }^{\circ} \mathrm{C}$. The features of $\mathrm{M} / \mathrm{G} / \mathrm{M}$ multiple emulsions are clearly seen at $20{ }^{\circ} \mathrm{C}$.
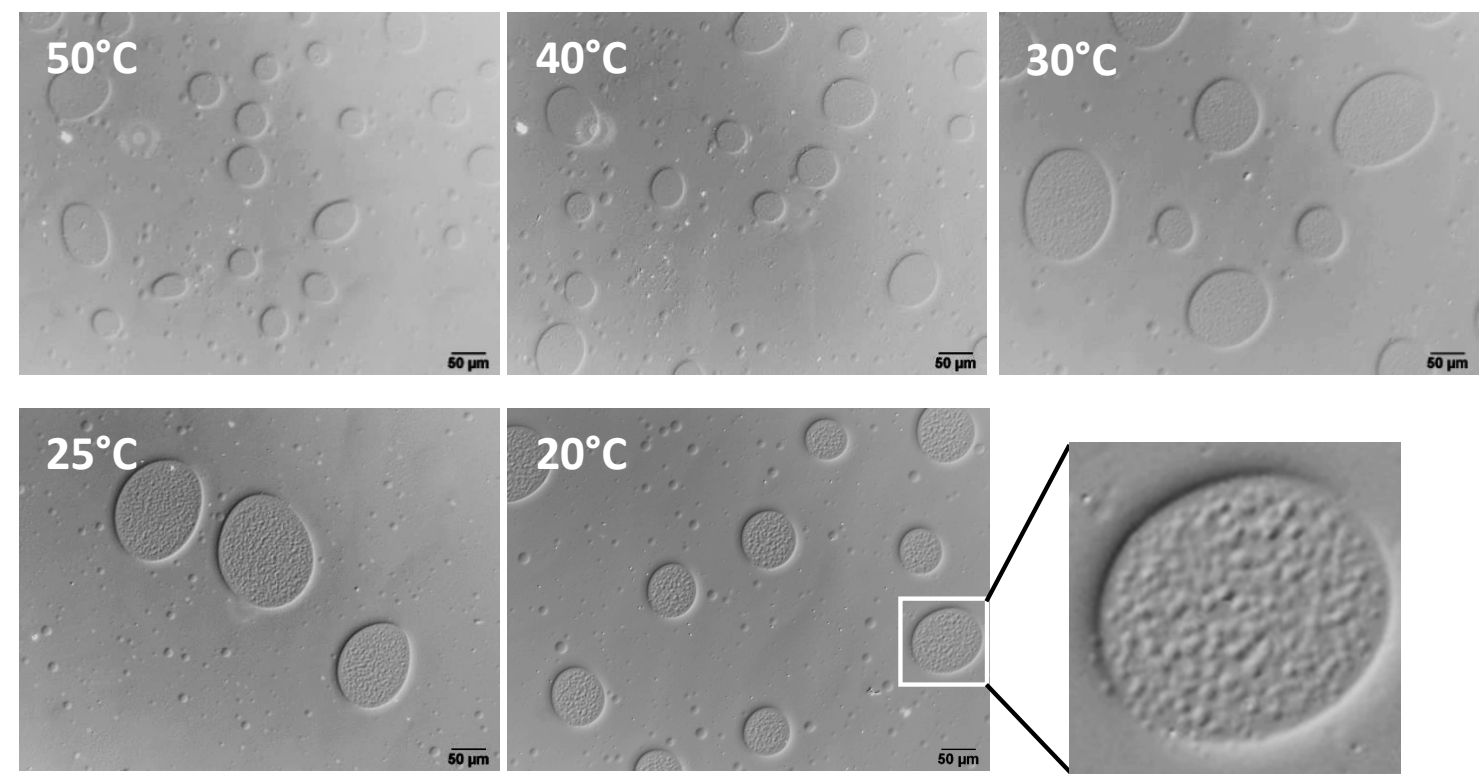

Fig. 7. Formation of multiple $\mathrm{M} / \mathrm{G} / \mathrm{M}$ emulsions, by slow cooling of a $\mathrm{G} / \mathrm{M}$ emulsion, from 50 to $20^{\circ} \mathrm{C}$. The overall composition was $4 \mathrm{wt} \%$ gelatin and $13 \mathrm{wt} \%$ maltodextrin, and cooling velocity was controlled at $\approx 2{ }^{\circ} \mathrm{C} / \mathrm{min}$, using a microscope sample holder with a thermostated hot stage. The inner droplets of multiple emulsions are clearly seen in the expanded image of a droplet at $20^{\circ} \mathrm{C}$. 
The precise temperature, at which condensation of internal droplets occurs, forming maltodextrin-in-gelatin $(\mathrm{M} / \mathrm{G})$ inner droplets, cannot be determined. Observations by optical microscopy indicated that it was a gradual process, with a threshold temperature difficult to be stablished.

The spontaneous formation of inner maltodextrin droplets could be due to several reasons. First, oversaturation of maltodextrin, inside the gelatin phase, probably leads to segregation of maltodextrin droplets. Second, another parameter that could play an important role is the variations in viscosity when temperature is reduced from 50 to $20^{\circ} \mathrm{C}$. For this reason, the gel point of gelatin was measured, by analysing the increase in viscosity when cooling down. As shown in Supporting Information (Fig. S4), gelation of $4 \mathrm{wt} \%$ gelatin occurs at $17.5^{\circ} \mathrm{C}$ (this concentration was selected because its use in the preparation of multiple emulsions). This result demonstrates that viscosity variations do not play any important role in the formation and stability of $\mathrm{M} / \mathrm{G} / \mathrm{M}$ multiple emulsions, since viscosity only presents a significant increase below $20^{\circ} \mathrm{C}$.

Consequently, concentration of maltodextrin is probably the key factor that most influences the appearance of inner droplets. As it was mentioned above, the formation of internal droplets inside gelatin-in-matodextrin larger drops was observed only in compositions that were near to the binodal line. This tendency is clearly shown in Fig. 8. The overall composition inside the external droplets is determined by the intersection of the tie line, with the binodal line. Emulsions with compositions lying further away from the tie line, have thus continuous and dispersed phases with higher gelatin or maltodextrin concentrations. As an example, a $4.5 \mathrm{wt} \%$ $\mathrm{G} / 11 \mathrm{wt} \% \mathrm{M}$ emulsion might have a dispersed phase of $\sim 10 \mathrm{wt} \% \mathrm{G} / 5 \mathrm{wt} \% \mathrm{M}$ and a continuous phase of $\sim 2 \mathrm{wt} \% \mathrm{G} / 14 \mathrm{wt} \% \mathrm{M}$; while a $4.5 \mathrm{wt} \% \mathrm{G} / 20 \mathrm{wt} \% \mathrm{M}$ emulsion could have a dispersed phase of $\sim 20 \mathrm{wt} \% \mathrm{G} / 5 \mathrm{wt} \% \mathrm{M}$ and a continuous phase of $\sim 0.5 \mathrm{wt} \% \mathrm{G} / 25$ $\mathrm{wt} \% \mathrm{M}$. At those high concentrations, temperature change may have a lower effect on phase behaviour, while in the first case a temperature change induces a shift of the binodal line and thus promotes formation of internal droplets.
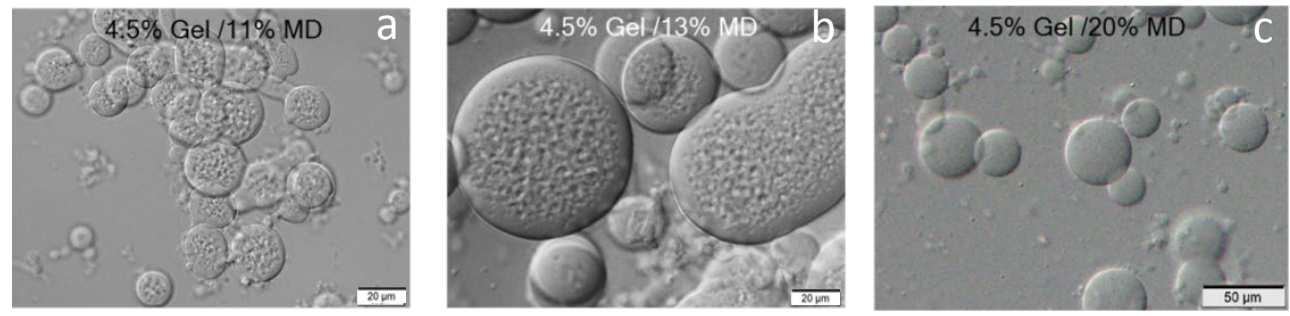

Fig. 8. Internal secondary droplets formation depending on the distance to the binodal line. Concentration of gelatin was kept constant at $4.5 \mathrm{wt} \%$, and maltodextrin concentration was varied from 11 to 20 wt\%.

It should be remarked that multiple $\mathrm{M} / \mathrm{G} / \mathrm{M}$ emulsions were obtained by reducing temperature at $1^{\circ} \mathrm{C} / \mathrm{min}$ or $2^{\circ} \mathrm{C} / \mathrm{min}$ cooling velocity. It could be presumed that a very fast cooling could lead to a metastable scenario without inner droplets, whereas a much slower cooling would allow diffusion of excess maltodextrin and formation of inner droplets would also not occur. Therefore, probably there is an optimal cooling rate. In any case, both 1 and $2{ }^{\circ} \mathrm{C} / \mathrm{min}$ provided good results, and consequently cooling velocity was not studied further. Anyway, these promising results illustrate that is possible to obtain multiple emulsions with small inner 
droplets and showing an enhanced stability, in comparison with multiple emulsions prepared by mechanical methods.

\section{Stabilization of $M / G / M$ emulsions, by addition of mucin}

To improve stability of multiple emulsions, it was necessary to add a compound which adsorbs in the interface. With this aim, mucin was used. This molecule is insoluble in aqueous media therefore; the mixture with gelatin and maltodextrin gives rise to the formation of a suspension. Initially, an optimal procedure to disperse homogeneously the mucin was carried out. Mucin particles were dispersed in water by vortex agitation, obtaining a particle size of $102.5 \mu \mathrm{m}$ (measured as $\mathrm{D}[4,3]$ ) and a polydispersity of 35.9 (calculated as the Span). Ultrasonication greatly reduced both particle size and polydispersity, achieving $2.9 \mu \mathrm{m}$ and 6.5 , respectively. The particle size distribution of the ultrasonicated sample is shown in Fig. S2 (Supporting Information).

In order to understand the influence of mucin addition on the stabilization of emulsions, $\mathrm{G} / \mathrm{M}$ simple emulsions were prepared with the same composition that would be used for the preparation of multiple emulsions ( $4 \mathrm{wt} \%$ gelatin and $13 \mathrm{wt} \%$ maltodextrin). First, the mucin suspension was added to the maltodextrin solution at $45{ }^{\circ} \mathrm{C}$, and this mixture was stirred for 15 min at $50{ }^{\circ} \mathrm{C}$ with a magnet at $500 \mathrm{rpm}$. Next, gelatin was added to those mucin-maltodextrin mixtures, applying vortex agitation for 20 seconds. The resulting emulsions, prepared with 0,1 and $2 \mathrm{wt} \%$ mucin, were kept at $50{ }^{\circ} \mathrm{C}$ during 10 days while being characterized by visual observation. The results (shown in Supporting Information, Fig. S5) indicate that addition of mucin increased emulsion stability, as it can be clearly seen by comparing the samples 3 hours after preparation. Emulsions with mucin presented a homogeneous aspect, whereas the sample without mucin showed an extensive phase separation. Moreover, in presence of mucin, emulsions were still present after 10 days, despite sedimentation. In conclusion, mucin particles were able to stabilize $\mathrm{G} / \mathrm{M}$ droplets. Then, the next step was evaluating if mucin particles could also stabilize multiple emulsions.

M/G/M multiple emulsions, stabilized with mucin, were prepared with $4 \mathrm{wt} \%$ gelatin and 13 $\mathrm{wt} \%$ maltodextrin, by first dispersing mucin in the maltodextrin phase at $45^{\circ} \mathrm{C}$. The mixture was stirred for $15 \mathrm{~min}$ at $45^{\circ} \mathrm{C}$ with a magnet at $500 \mathrm{rpm}$. Then, gelatin was added to those mucinmaltodextrin mixtures, applying vortex agitation for 20 seconds. Finally, Then, M/G/M multiple emulsions were formed by cooling down from $45^{\circ} \mathrm{C}$ to room temperature with a cooling rate of $1{ }^{\circ} \mathrm{C} / \mathrm{min}$ 
Different mucin concentrations were studied, from 0 to $2 \mathrm{wt} \%$ and their stability was assessed over time, by visual observations and optical microscopy.

Just after preparation and 10 days later, , samples were characterized by visual observation and optical microscopy without mucin and in presence of mucin, at 1 and $2 \mathrm{wt} \%$ concentrations (Fig. 9). As it can be seen, the addition of mucin produces an increase of sample turbidity.

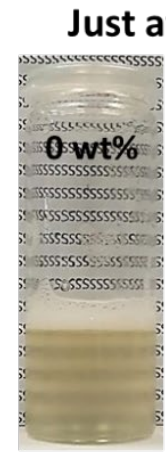

$0 \mathrm{wt} \%$
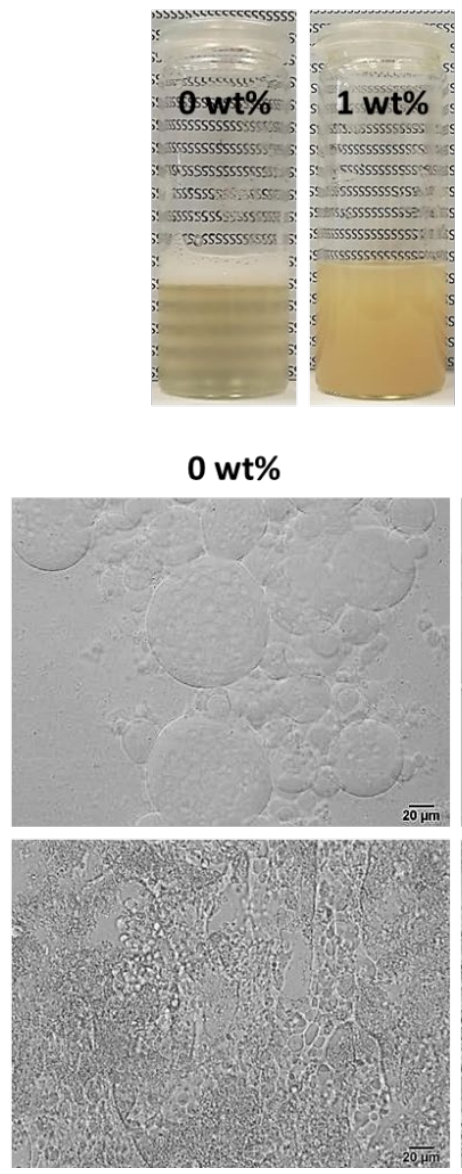

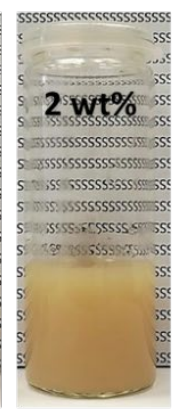

1 wt\% mucin
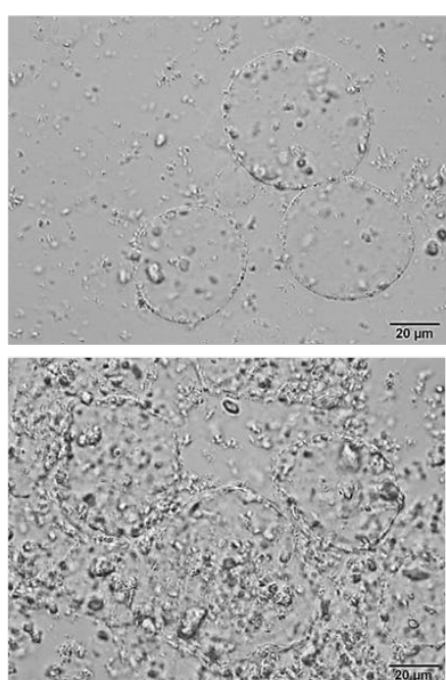

10 days after

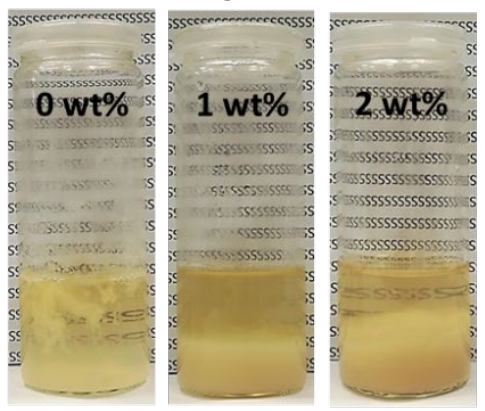

2 wt\% mucin
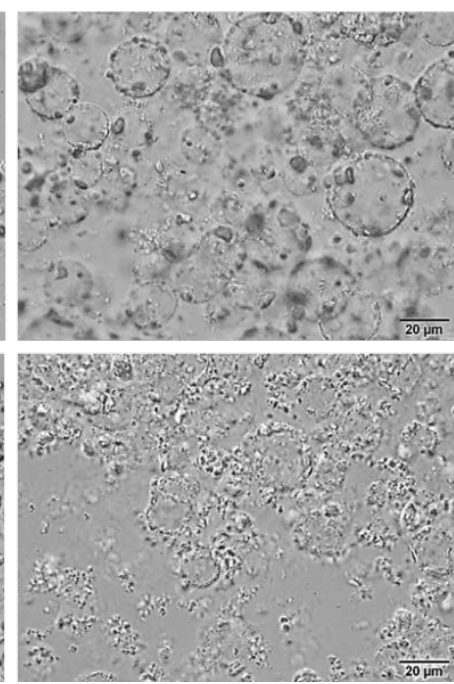

Fig. 9. Stability of multiple $\mathrm{M} / \mathrm{G} / \mathrm{M}$ emulsions as a function of mucin particle concentration, from 0 to 2 $w t \%$, at $25^{\circ} \mathrm{C}$. The visual aspect of freshly prepared samples and those after 10 days are shown.

Images in Fig. 9 clearly show that mucin particles greatly improve stability of M/G/M multiple emulsions, with just $1 \mathrm{wt} \%$ mucin. In absence of mucin particles, the emulsion is quite unstable, since the initial emulsion is not observed after 10 days at $25^{\circ} \mathrm{C}$. When micrographs are compared as a function of mucin concentration, one can observe a decrease of droplet size by increasing mucin concentration, from $30-60 \mu \mathrm{m}$ without mucin to $\approx 20 \mu \mathrm{m}$ at $2 \mathrm{wt} \%$ mucin. This variation of droplet size illustrates the Pickering effect of mucin particles due to their adsorption on the interface. After 10 days, droplet size does not show important variations of droplet size and mucin particles still remain on the interface.

Regarding viscosity of samples, no large differences were visually observed. This was expected, since temperature $\left(25^{\circ} \mathrm{C}\right)$ was above the gelation point of gelatin (around $17.5^{\circ} \mathrm{C}$ ) and the 
concentration of mucin particles (up to $2 \mathrm{wt} \%$ ) was not high enough to increase viscosity. Therefore, the improved stability can only be attributed to particle adsorption on interfaces.

It can also be observed that mucin particles tend to aggregate, but these aggregates also remain adsorbed on the interface. Summarizing, it should be remarked that these images illustrate the presence of droplets, stable after 10 days because of mucin adsorbed on interfaces. However, the inner smaller droplets were not visible. This was attributed to the high transparency of multiple M/G/M droplets, and more detailed images were searched by labelling with fluorescent dyes.

$\mathrm{M} / \mathrm{G} / \mathrm{M}$ multiple emulsions were observed by various optical microscopy techniques, using various labelling methods. First, emulsions with $2 \mathrm{wt} \%$ mucin and without labelling were observed by interference contrast microscopy, enhancing visibility of particles with higher refractive index (Fig. 10a). This image confirms that surface of $M / G$ multiple droplets is covered with adsorbed mucin particles. However, most mucin particles were not adsorbed, and a large number of particles seemed to floating around the external maltodextrin phase. Microscopy observations also indicated that $1 \mathrm{wt} \%$ mucin was perhaps the optimum amount, and further characterization was performed with such mucin quantity.

Fig. 10b-e show images acquired using fluorescence filters, in a sample with $1 \mathrm{wt} \%$ mucin. The picture in Fig. 10b was captured by fluorescence optical microscopy when RBITC was previously used to label mucin. This image clearly shows that most mucin particles are adsorbed on the surface of $\mathrm{M} / \mathrm{G}$ droplets, but some particles seem to be inside $\mathrm{M} / \mathrm{G}$ droplets and other are just floating in the external $\mathrm{M}$ phase. The inner maltodextrin droplets, inside gelatin droplets were observed by labelling maltodextrin with FITC (Fig. 10c). Maltodextrin inner droplets are seen as green spots inside gelatin droplets that appear black. The external maltodextrin phase is also seen as green.

Laser Scanning Confocal Optical Microscopy allows the localization in optical sections of two different fluorescence labelling agents. In presence of maltodextrin labelled with FITC and mucin labelled with RBITC (Fig. 10d) most of mucin particles are adsorbed on the surface of $\mathrm{M} / \mathrm{G}$ droplets, and thus, confirming mucin ability to adsorb on gelatin/maltodextrin interface. In addition, inner maltodextrin droplets can also be observed, confirming once again the nature of $\mathrm{M} / \mathrm{G} / \mathrm{M}$ multiple emulsions.

Finally, a 3D reconstruction was performed by confocal microscopy of M/G/M multiple emulsions in which gelatin was labelled with FITC and mucin with RBITC. The example displayed in Fig. 10e shows that the coverage of gelatin droplets by mucin particles is rather incomplete, with many uncovered patches. Nevertheless, it should be remarked that this configuration is still able to provide a good colloidal stability, as demonstrated in Fig. 9. 

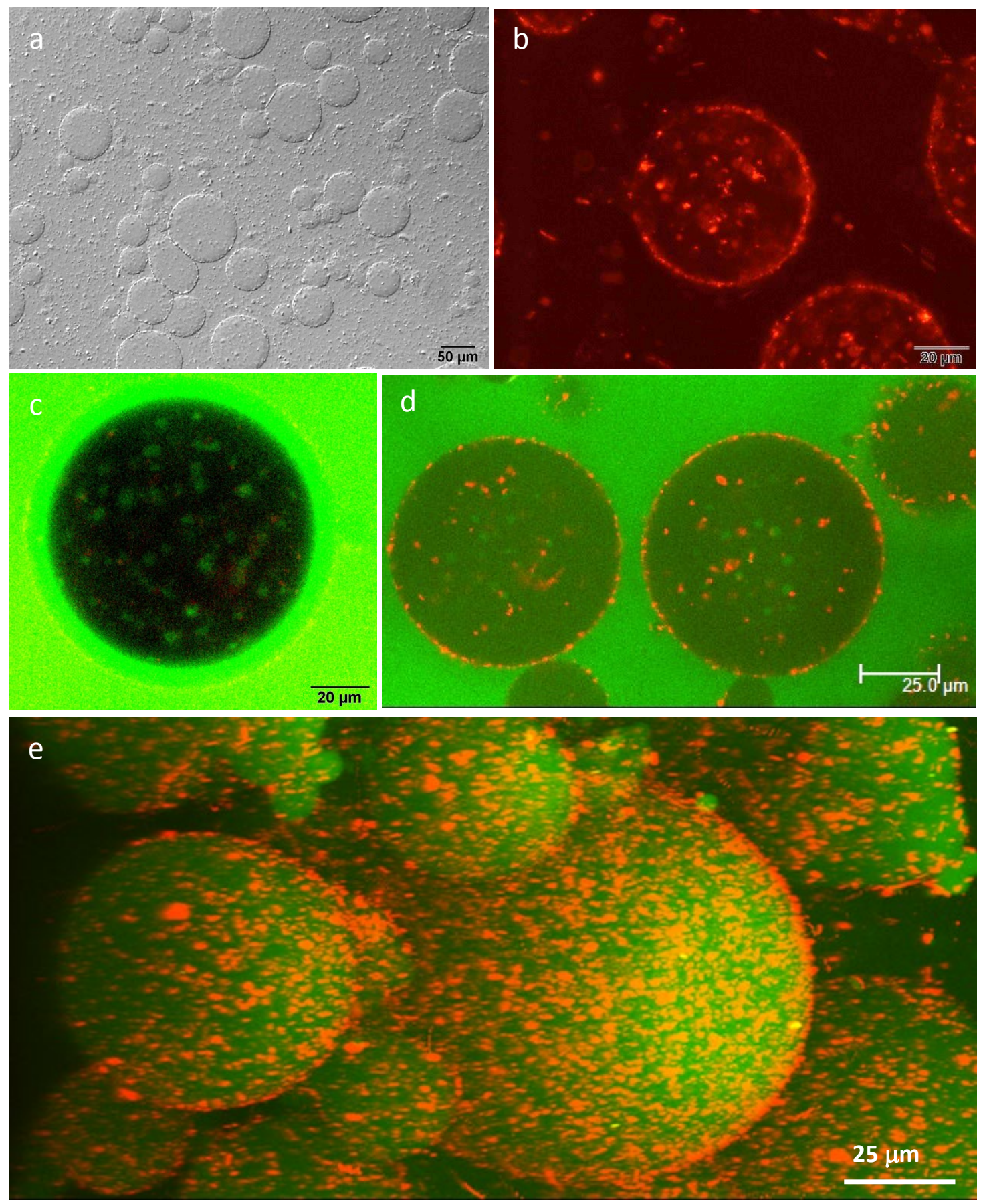

Fig. 10. Illustrative examples of optical microscopy images of $M / G / M$ multiple emulsions. (a) Emulsion prepared with 4 wt\% gelatin, 13 wt\% maltodextrin and 2 wt\% mucin; (b) 4 wt\% gelatin, 13 wt\% maltodextrin and 1 wt\% RBITC-labelled mucin; (c) 4 wt\% gelatin, 13 wt\% FITC-labelled maltodextrin and 1 wt\% RBITC-labelled mucin; (d) Same composition as (c), observed with a Laser Scanning Confocal Microscope; (e) 3D software-assisted reconstruction of LSCM images. 
It has been demonstrated that the multiple emulsions were formed above the gel point of gelatin, which indicated that the viscosity did not influence in the stability of emulsions and consequently, the main contribution to emulsion stability comes from mucin particles adsorbed on $\mathrm{W} / \mathrm{W}$ interface. Therefore, $\mathrm{M} / \mathrm{G} / \mathrm{M}$ multiple emulsions are stable because of adsorption of mucin on the G-M interface, stabilising the $\mathrm{M} / \mathrm{G}$ droplets in the multiple emulsions.

In conclusion, it has been demonstrated that $\mathrm{M} / \mathrm{G} / \mathrm{M}$ multiple emulsions are stable. The precise mechanism of stability remains to be fully understood, but it is clear that $\mathrm{G} / \mathrm{M}$ droplets dispersed in maltodextrin, forming $\mathrm{M} / \mathrm{G} / \mathrm{M}$ emulsion, are stabilized by the presence of mucin particles adsorbed on the $\mathrm{G} / \mathrm{M}$ interface. Regarding the smaller $\mathrm{M} / \mathrm{G}$ droplets, located inside the gelatin larger drops, its stability is more difficult to assess because of its smaller size and difficult observations. However, the evidence indicates that these smaller M/G droplets are also quite stable, despite the fact that these droplets have little presence of adsorbed mucin, and that gelatin is not gelled above $25^{\circ} \mathrm{C}$.

\section{Conclusions}

Visual observations clearly reflect a straightforward relation between the method of emulsion preparation and emulsion properties. M/G single emulsions prepared by phase transition showed smaller droplets with higher stability. The transition method based on $\mathrm{pH}$ change has allowed obtaining primary emulsions with droplet sizes around 5-10 $\mu \mathrm{m}$. This is an important reduction in droplet size, in comparison to results obtained by mechanical agitation, $\approx 20-40 \mu \mathrm{m}$. Moreover, emulsions prepared by phase transition showed an improvement in colloidal stability.

Dropwise addition of a primary $\mathrm{M} / \mathrm{G}$ emulsion, on a maltodextrin aqueous solution, allowed formulation of multiple $\mathrm{M} / \mathrm{G} / \mathrm{M}$ emulsion and demonstrated that multiple $\mathrm{W} / \mathrm{W} / \mathrm{W}$ emulsions can be prepared by two-step emulsification processes, similarly to conventional $\mathrm{W} / \mathrm{O} / \mathrm{W}$ or $\mathrm{O} / \mathrm{W} / \mathrm{O}$ emulsions. Phase transitions, either by changing $\mathrm{pH}$ or modifying temperature, can be applied to assist emulsification. M/G/M multiple emulsions can be obtained by cooling down single $\mathrm{G} / \mathrm{M}$ emulsions, in compositions close to the binodal boundary line. Finally, colloidal stability of $\mathrm{M} / \mathrm{G} / \mathrm{M}$ drops was greatly improved by adding $1 \mathrm{wt} \%$ mucin particles, which adsorbed on the $\mathrm{G} / \mathrm{M}$ interface.

Three interesting conclusions can be drawn: (a) Either $\mathrm{M} / \mathrm{G}$ or $\mathrm{G} / \mathrm{M}$ emulsions can be prepared by phase transition methods, from one single phase $\mathrm{L}$, to two equilibrium phases $\mathrm{L}_{1}+\mathrm{L}_{2}$, inducing oversaturation of one phase that condenses forming droplets; (b) Multiple M/G/M emulsions can be obtained by a simple and cost-effective emulsification method, which is not based on microfluidics; and (c) Mucin glycoprotein particles are effective stabilizers for G/M emulsions, in which mucin particles adsorb on the interface of gelatin-rich droplets dispersed in a maltodextrin-rich aqueous solution.

To our knowledge, this is the first report that describes the formation of multiple W/W/W Pickering emulsions without using microfluidic devices, and it is also the first time that mucin particles are used to stabilize all-aqueous emulsions. W/W/W multiple emulsions can be prepared applying simple and easy formulation paths. This breakthrough in the formation and stabilization of multiple W/W/W Pickering emulsions certainly encourages deeper studies. The observed results could be extrapolated to other mixtures of proteins and polysaccharides, given 
that emulsion formation can be controlled making use of information provided by phase diagrams. Indeed, the strategies described here could lead to a better control of all-aqueous emulsions, with potential future applications in many technological or biomedical fields.

\section{Associated Content}

Supporting Information. Fig. S1. Phase separation of an aqueous mixture of $12 \mathrm{wt} \%$ gelatin and $6 \mathrm{wt} \%$ maltodextrin, kept at $50^{\circ} \mathrm{C}$ during 5 days days; Fig. S2: Phase separation of maltodextrin/gelatin/maltodextrin multiple emulsion, in absence of mucin particles, after $3 \mathrm{~h}$ at $37^{\circ}$ C.; Fig. S3: Particle size distribution of mucin particles, used as stabilizer; Fig. S4. Determination of the gel point for gelatin solution at $4 \mathrm{wt} \%$; Fig. S5. Stability of emulsion with composition $4 \mathrm{wt} \%$ gelatin and $13 \mathrm{wt} \%$ maltodextrin kept at $50^{\circ} \mathrm{C}$ during 10 days.

\section{Acknowledgements}

The authors greatly acknowledge the financial support from the Spanish Ministry of Economy and Competitiveness (CTQ2017-84998-P and CTQ2016-80645-R projects) and Marie Sklodowska Curie Initial Training Networks (FP7-PEOPLE-2013-ITN-606713, BIBAFOODS project). Support from Generalitat de Catalunya (2017SGR1778 and TECCIT15-1-0009) and Centro de Investigación Biomédica en Red de Bioingeniería, Biomateriales y Nanomedicina (CIBER-BBN), is also acknowledged. The authors also acknowledge the "Grupo de Nanotecnología Farmacéutica", of the University of Barcelona (UB), in the Faculty of Pharmacy, which forms an Associated Unit to CSIC. Authors are also very grateful to the European Regional Development Fund (Fondo Europeo de Desarrollo Regional, FEDER), which provides support to these government programs.

Characterization, except Confocal Microscopy, have been performed by the Nanostructured Liquid Characterization Unit, located at the Institute of Advanced Chemistry of Catalonia (IQAC), belonging to the Spanish National Research Council (CSIC) and affiliated to the NANBIOSIS ICTS of the Biomedical Networking Center (CIBER-BBN), which is financed by Instituto de Salud Carlos III. Laser Confocal Optical Microscopy was performed at the Advanced Optical Microscopy Unit of the Scientific and Technological Centres of University of Barcelona.

The authors also greatly thank Carlos Rodríguez-Abreu and Conxita Solans (IQAC-CSIC), and Allan Mackie and Josep Bonet (Universitat Rovira i Virgili, Spain) for very useful discussions.

Declarations of interest: none.

\section{References}

(1) Esquena, J. Water-in-Water (W/W) Emulsions. Curr. Opin. Colloid Interface Sci. 2016, 25, 109-119.

(2) Beijerinck, M. W. Ueber Eine Eigentümlichkeit Der Löslichen Stärke. Zentralblatt für Bakteriol. Parasitenkd. und Infekt. 1896, 2 (22), 697-699. 
(3) Hatti-Kaul, R. Aqueous Two-Phase Systems: A General Overview. Mol. Biotechnol. 2001, 19, $269-277$.

(4) Grinberg, V. Y.; Tolstoguzov, V. B. Thermodynamic Incompatibility of Proteins and Polysaccharides in Solutions. Food Hydrocoll. 1997, 11 (2), 145-158.

(5) Doublier, J. L.; Garnier, C.; Renard, D.; Sanchez, C. Protein-Polysaccharide Interactions. Curr. Opin. Colloid Interface Sci. 2000, 5 (3-4), 202-214.

(6) Clark, A. H. Direct Analysis of Experimental Tie Line Data (Two Polymer - One Solvent Systems) Using Flory - Huggins Theory. Carbohydr. Polym. 2000, 42, 337-351.

(7) McClements, D. Nanoparticle-and Microparticle-Based Delivery Systems; CRC Press, 2014.

(8) Semenova, M. G.; Dickinson, E. Biopolymers in Food Colloids: Thermodynamics and Molecular Interactions; 1st Edition, CRC Press: London, 2010.

(9) Scholten, E.; Sagis, L. M. C.; Van Linden, E. Der. Effect of Bending Rigidity and Interfacial Permeability on the Dynamical Behavior of Water-in-Water Emulsions. J. Phys. Chem. B 2006, 110 (7), 3250-3256.

(10) Sagis, L. M. C. Fluctuations of Permeable Interfaces in Water-in-Water Emulsions. 2007, 98 (6).

(11) Vis, M.; Peters, V. F. D.; Blokhuis, E. M.; Lekkerkerker, H. N. W.; Erné, B. H.; Tromp, R. H. Effects of Electric Charge on the Interfacial Tension between Coexisting Aqueous Mixtures of Polyelectrolyte and Neutral Polymer. Macromolecules 2015, 48, 7335-7345.

(12) Vis, M.; Peters, V. F. D.; Tromp, R. H.; Erne, B. H. Donnan Potentials in Aqueous Phase-Separated Polymer Mixtures. Langmuir 2014, 30, 5755-5762.

(13) Vis, M.; Erné, B. H.; Tromp, R. H. Chemical Physics of Water-Water Interfaces. Biointerphases 2016, 11 (1), $1-8$.

(14) Vis, M.; Peters, V. F. D.; Erné, B. H.; Tromp, R. H. Ion Entropy in Phase-Separated Aqueous Mixtures of Polyelectrolyte and Neutral Polymer. Macromolecules 2015, 48 (8), 2819-2828.

(15) Norton, I. T. T.; Frith, W. J. J. Microstructure Design in Mixed Biopolymer Composites. Food Hydrocoll. 2001, 15 (4-6), 543-553.

(16) Ding, P.; Wolf, B.; Frith, W. J.; Clark, A. H.; Norton, I. T.; Pacek, A. W. Interfacial Tension in PhaseSeparated Gelatin/Dextran Aqueous Mixtures. J. Colloid Interface Sci. 2002, 253 (2), 367-376.

(17) Scholten, E.; Visser, J. E.; Sagis, L. M. C.; van der Linden, E. Ultralow Interfacial Tensions in an Aqueous Phase-Separated Gelatin/Dextran and Gelatin/Gum Arabic System: A Comparison. Langmuir 2004, 20 (6), 2292-2297.

(18) Vis, M.; Peters, V. F. D.; Blokhuis, E. M.; Lekkerkerker, H. N. W.; Erné, B. H.; Tromp, R. H. Decreased Interfacial Tension of Demixed Aqueous Polymer Solutions Due to Charge. Phys. Rev. Lett. 2015, 115 (7), 078303.

(19) Vis, M.; Blokhuis, E. M.; Erné, B. H.; Tromp, R. H.; Lekkerkerker, H. N. W. Interfacial Tension of PhaseSeparated Polydisperse Mixed Polymer Solutions. J. Phys. Chem. B 2018, 122 (13), 3354-3362.

(20) Cheung Shum, A. H.; Varnell, J.; Weitz, D. A. Microfluidic Fabrication of Water-in-Water (w/w) Jets and Emulsions. Biomicrofluics 2012, 6 (1), 12808.

(21) Moon, B. U.; Abbasi, N.; Jones, S. G.; Hwang, D. K.; Tsai, S. S. H. Water-in-Water Droplets by Passive Microfluidic Flow Focusing. Anal. Chem. 2016, 88 (7), 3982-3989.

(22) Zhou, C.; Zhu, P.; Tian, Y.; Tang, X.; Shi, R.; Wang, L. Microfluidic Generation of Aqueous Two-PhaseSystem (ATPS) Droplets by Oil-Droplet Choppers. Lab Chip 2017, 17 (19), 3310-3317.

(23) Song, Y.; Sauret, A.; Shum, H. C. All-Aqueous Multiphase Microfluidics. Biomicrofluidics 2013, 7 (6), 1 12.

(24) Arriaga, L. R.; Datta, S. S.; Kim, S.-H.; Amstad, E.; Kodger, T. E.; Monroy, F.; Weitz, D. A. Ultrathin Shell Double Emulsion Templated Giant Unilamellar Lipid Vesicles with Controlled Microdomain Formation. Small 2014, 10 (5), 950-956.

(25) Yasukawa, M.; Kamio, E.; Ono, T. Monodisperse Water-in-Water-in-Oil Emulsion Droplets. 2011, 12 (2), 263-266.

(26) Poortinga, A. T. Microcapsules from Self-Assembled Colloidal Particles Using Aqueous Phase-Separated Polymer Solutions. Langmuir 2008, 24 (5), 1644-1647.

(27) Dickinson, E. Particle-Based Stabilization of Water-in-Water Emulsions Containing Mixed Biopolymers. Trends Food Sci. Technol. 2019, 83, 31-40.

(28) Firoozmand, H.; Murray, B. S.; Dickinson, E. Interfacial Structuring in a Phase-Separating Mixed Biopolymer Solution Containing Colloidal Particles. Langmuir 2009, 25 (3), 1300-1305.

(29) Balakrishnan, G.; Nicolai, T.; Benyahia, L.; Durand, D. Particles Trapped at the Droplet Interface in Waterin-Water Emulsions. Langmuir 2012, 28, 5921-5926.

(30) Nguyen, B. T.; Nicolai, T.; Benyahia, L. Stabilization of Water-in-Water Emulsions by Addition of Protein Particles. Langmuir 2013, 29 (34), 10658-10664.

(31) Murray, B. S.; Phisarnchananan, N. Whey Protein Microgel Particles as Stabilizers of Waxy Corn Starch + Locust Bean Gum Water-in-Water Emulsions. Food Hydrocoll. 2016, 56, 161-169.

(32) Chatsisvili, N.; Philipse, A. P.; Loppinet, B.; Tromp, R. H. Colloidal Zein Particles at Water-Water Interfaces. Food Hydrocoll. 2017, 65, 17-23.

(33) Das, A. A. K.; Filby, B. W.; Geddes, D. A.; Legrande, D.; Paunov, V. N. High Throughput Fabrication of Cell Spheroids by Templating Water-in-Water Pickering Emulsions. Mater. Horizons 2017, 4 (6), 11961200.

(34) Binks, B. P.; Shi, H. Phase Inversion of Silica Particle-Stabilized Water-in-Water Emulsions. Langmuir 2019, 35 (11), 4046-4057.

(35) Murray, B. S.; Phisarnchananan, N. The Effect of Nanoparticles on the Phase Separation of Waxy Corn 
Starch + Locust Bean Gum or Guar Gum. Food Hydrocoll. 2014, 42, 92-99.

(36) Vis, M.; Opdam, J.; Van't Oor, I. S. J.; Soligno, G.; Van Roij, R.; Tromp, R. H.; Erné, B. H. Water-in-Water Emulsions Stabilized by Nanoplates. ACS Macro Lett. 2015, 4 (9), 965-968.

(37) Ganley, W. J.; Ryan, P. T.; van Duijneveldt, J. S. Stabilisation of Water-in-Water Emulsions by Montmorillonite Platelets. J. Colloid Interface Sci. 2017, 505, 139-147.

(38) Peddireddy, K. R.; Nicolai, T.; Benyahia, L.; Capron, I. Stabilization of Water-in-Water Emulsions by Nanorods. 2016, 5 (3), 283-286.

(39) Ayed, E. B.; Cochereau, R.; Dechancé, C.; Capron, I.; Nicolai, T.; Benyahia, L. Water-in-Water Emulsion Gels Stabilized by Cellulose Nanocrystals. Langmuir 2018, 34, 6887-6893.

(40) Gonzalez-Jordan, A.; Nicolai, T.; Benyahia, L. Influence of the Protein Particle Morphology and Partitioning on the Behavior of Particle-Stabilized Water-in-Water Emulsions. 2016, 32 (28), 7189-7197.

(41) Nicolai, T.; Murray, B. Particle Stabilized Water in Water Emulsions. Food Hydrocoll. 2017, 68, 157-163.

(42) Gonzalez-Jordan, A.; Nicolai, T.; Benyahia, L. Enhancement of the Particle Stabilization of Water-in-Water Emulsions by Modulating the Phase Preference of the Particles. J. Colloid Interface Sci. 2018, 530, 505510.

(43) de Freitas, R. A.; Nicolai, T.; Chassenieux, C.; Benyahia, L. Stabilization of Water-in-Water Emulsions by Polysaccharide-Coated Protein Particles. Langmuir 2016, 32, 1227-1232.

(44) Capron, I.; Costeux, S.; Djabourov, M. Water in Water Emulsions: Phase Separation and Rheology of Biopolymer Solutions. Rheol. Acta 2001, 40 (5), 441-456.

(45) Singh, P.; Medronho, B.; Miguel, M. G.; Esquena, J. On the Encapsulation and Viability of Probiotic Bacteria in Edible Carboxymethyl Cellulose-Gelatin Water-in-Water Emulsions. Food Hydrocoll. 2018, 75, 41-50.

(46) Beldengrün, Y.; Aragon, J.; Prazeres, S. F.; Montalvo, G.; Miras, J.; Esquena, J. Gelatin/Maltodextrin Water-in-Water (W/W) Emulsions for the Preparation of Cross-Linked Enzyme-Loaded Microgels. Langmuir 2018, 34 (33), 9731-9743.

(47) Kasapis, S.; Morris, E. R.; Norton, I. T.; Clark, A. H. Phase Equilibria and Gelation in Gelatin/Maltodextrin Systems - Part I: Gelation of Individual Components. Carbohydr. Polym. 1993, 21 (4), 243-248.

(48) Kasapis, S.; Morris, E. R.; Norton, I. T.; Clark, A. H. Phase Equilibria and Gelation in Gelatin/Maltodextrin Systems - Part IV: Composition-Dependence of Mixed-Gel Moduli. Carbohydr. Polym. 1993, 21 (4), 269 276.

(49) Alevisopoulos, S.; Kasapis, S.; Abeysekera, R. Formation of Kinetically Trapped Gels in the Maltodextringelatin System. Carbohydr. Res. 1996, 293 (1), 79-99.

(50) Tyowua, A. T.; Yiase, S. G.; Binks, B. P. Double Oil-in-Oil-in-Oil Emulsions Stabilised Solely by Particles. J. Colloid Interface Sci. 2017, 488, 127-134.

(51) Sauret, A.; Shum, H. C. Forced Generation of Simple and Double Emulsions in All-Aqueous Systems. Appl. Phys. Lett. 2012, 100 (15).

(52) Song, Y.; Shum, H. C. Monodisperse w/w/w Double Emulsion Induced by Phase Separation. Langmuir 2012, 28 (33), 12054-12059.

(53) Beldengrün, Y. Water-in-Water Emulsions for Obtaining Enzyme-Loaded Microgels and Encapsulated Emulsions, University of Barcelona, 2018.

(54) Monroy, F.; Moleiro, L. H.; Ortega, S. A.; Arriaga, L. R. Microfluidic-Assisted Determination of Phase Diagrams of Aqueous Two-Phase Systems within Double Emulsion Drops. Present. 31th Eur. Colloids Interface Soc. (ECIS), Madrid, Spain. 2017.

(55) Kasapis, S.; Morris, E. R.; Norton, I. T.; Gidley, M. J. Phase Equilibria and Gelation in Gelatin/Maltodextrin Systems - Part II: Polymer Incompatibility in Solution. Carbohydr. Polym. 1993, 21 (4), 249-259.

(56) Kasapis, S.; Morris, E. R.; Norton, I. T.; Brown, C. R. T. Phase Equilibria and Gelation in Gelatin/Maltodextrin Systems - Part III: Phase Separation in Mixed Gels. Carbohydr. Polym. 1993, 21 (4), 261-268.

(57) de Belder, A. N.; Granath, K. Preparation and Properties of Fluorescein-Labelled Dextrans. Carbohydr. Res. 1973, 30 (2), 375-378.

(58) Lorén, N.; Hermansson, A.-M.; Williams, M. A. K.; Lundin, L.; Foster, T. J.; Hubbard, C. D.; Clark, A. H.; Norton, I. T.; Bergström, E. T.; Godall, D. M. Phase Separation Induced by Conformational Ordering of Gelatin in Gelatin/Maltodextrin Mixtures. Macromolecules 2001, 34, 289-297.

(59) Masullo, F.; Beldengrün, Y.; Miras, J.; Mackie, A. D.; Esquena, J.; Bonet-Avalos, J. Phase Behavior of Gelatin/Maltodextrin Aqueous Mixtures Studied from a Combined Experimental and Theoretical Approach. (Unpublished Work. 2019.

(60) Hoey, A.; Ryan, J. T.; Fitzsimons, S. M.; Morris, E. R. Segregative Interactions in Single-Phase Mixtures of Gelling (Potato) Maltodextrin with Other Hydrocolloids. In Gums and Stabilisers for the Food Industry 18: Hydrocolloid Functionality for Affordable and Sustainable Global Food Solutions; Williams, P. A., Phillips, G., Eds.; Royal Society of Chemistry, 2016; pp 305-312.

(61) Cates, M. E.; Clegg, P. S. Bijels: A New Class of Soft Materials. Soft Matter 2008, 4 (11), 2132-2138.

(62) Lorén, N.; Langton, M.; Hermansson, A. M. Confocal Laser Scanning Microscopy and Image Analysis of Kinetically Trapped Phase-Separated Gelatin/Maltodextrin Gels. Food Hydrocoll. 1999, 13, 185-198. 